\title{
Feshbach-Fano approach for calculation of Auger decay rates using equation-of-motion coupled-cluster wave functions: Theory and implementation
}

\author{
Wojciech Skomorowski* and Anna I. Krylov ${ }^{\dagger}$ \\ Department of Chemistry, University of Southern California, Los Angeles, California 90089, USA
}

\begin{abstract}
X-ray absorption creates electron vacancies in the core shell. These highly excited states often relax by Auger decay - an autoionization process in which one valence electron fills the core hole and another valence electron is ejected into the ionization continuum. Despite the important role of Auger processes in many experimental settings, their first-principle modeling is challenging, even for small systems. The difficulty stems from the necessity to describe many-electron continuum (unbound) states, which cannot be tackled with standard quantum-chemistry methods. We present a novel approach to calculate Auger decay rates by combining Feshbach-Fano resonance theory with the equation-of-motion coupled-cluster (EOM-CCSD) framework. We use the core-valence separation (CVS) scheme to define projectors into the bound (square-integrable) and unbound (continuum) subspaces of the full function space. The continuum many-body decay states are represented by products of an appropriate EOM-CCSD state and a free-electron state, described by a continuum orbital. The Auger rates are expressed in terms of reduced quantities, two-body Dyson amplitudes (objects analogous to the two-particle transition density matrix), contracted with two-electron bound-continuum integrals. Here we consider two approximate treatments of the free electron: a plane wave and a Coulomb wave with an effective charge, which allow us to evaluate all requisite integrals analytically; however, the theory can be extended to incorporate more sophisticated description of the continuum orbital.
\end{abstract}

\section{INTRODUCTION}

Owing to their ability to target specific atomic sites while being sensitive to the chemical environment, corelevel spectroscopies are powerful tools for interrogating molecular structure[1-3]. The underlying versatile selection rules governing excitation processes to the excited states in either bound or continuum part of the spectrum enable a broad range of applications. Advances in laser technology and the development of novel $\mathrm{X}$-ray sources have opened up a new area of applications in which core-level spectroscopies can be used as probes to study electron and nuclear dynamics with unprecedented time and space resolution [4-9]. Recently, $\mathrm{X}$-ray spectroscopy was used to reveal the dynamics of liquid $\mathrm{H}_{2} \mathrm{O}^{+}[10,11]$, a photo-induced ring-opening reaction [12], charge-migration and charge-transfer reactions [13-15], and to interrogate an interplay between open-shell spin-coupling and Jahn-Teller distortion in the benzene radical cation $[16,17]$.

Absorption of an X-ray photon, creating a vacancy in the core shell, leaves the molecule in a highly excited state. In molecules composed of light atoms (such as $\mathrm{C}$, N, or $\mathrm{O}$ ), these core-level states decay predominantly through a non-radiative autoionization process called Auger decay [18]. In this process, shown schematically in Fig. 1, the core hole is filled with an electron from a valence orbital, liberating sufficient energy to eject another valence electron (called an Auger electron)

\footnotetext{
* skomorow@usc.edu

† krylov@usc.edu
}

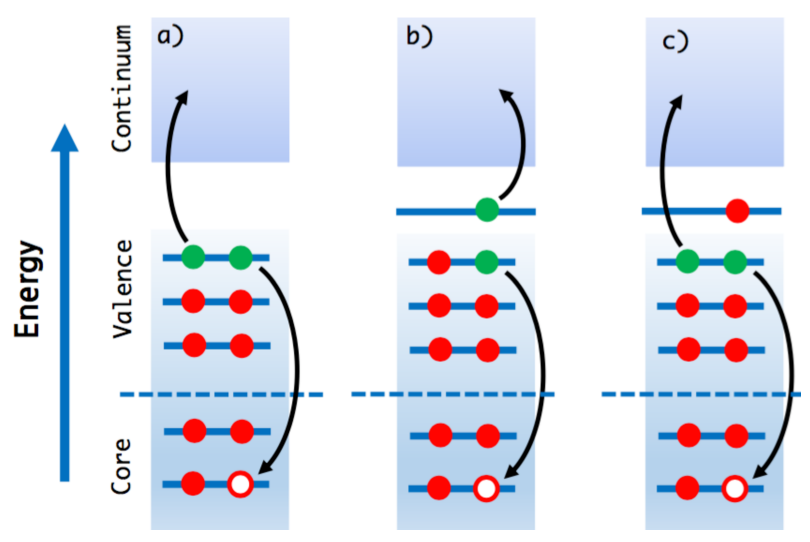

FIG. 1. Different types of Auger effect: (a) regular Auger decay, (b) resonant (participator) decay, and (c) resonant (spectator) decay. Regular Auger decay is relevant for Xray photoionization spectroscopy (XPS), whereas resonant Auger processes occur in X-ray absorption spectroscopy (XAS).

into the continuum. Having characteristic timescale on the order of femtoseconds, Auger processes compete directly with electron and nuclear dynamics triggered by prior X-ray photon absorption. Auger decay also plays an important role in molecular imaging using ultrashort X-ray pulses from free-electron lasers, where it contributes to the damage of the sample, limiting the achievable resolution $[19,20]$. By measuring the kinetic energy of the emitted electron, Auger electron spectroscopy is used in studies of surfaces, materials, nanostructures, and gas-phase molecules [21-24].

The first theoretical description of Auger decay was 
due to Wentzel, who employed a pertubative approach to calculate transition rates into the continuum in an atom with two active electrons [25]. The key assumption was that Auger decay is a two-step process in which the emission of the Auger electron is independent from the preceding core-shell depletion created by means of $\mathrm{X}$-ray photoionization or absorption transition. Hence, the initial state for the Auger decay can be treated as an electronically metastable state, undergoing spontaneous ionization.

Multichannel resonance scattering theory $[18,26]$ provides an alternative, more sophisticated treatment of autoionization, including the Auger effect. In this framework, the autoionizing state appears as a pole in the scattering matrix for complex-valued electron collision energy. The scattering wave function in the vicinity of an isolated resonance state can be decomposed into two parts: bound-like and continuum-like. The former is square-integrable and it closely resembles a regular bound state, whereas the latter is non-square integrable and fully determines the asymptotic behavior of the state.

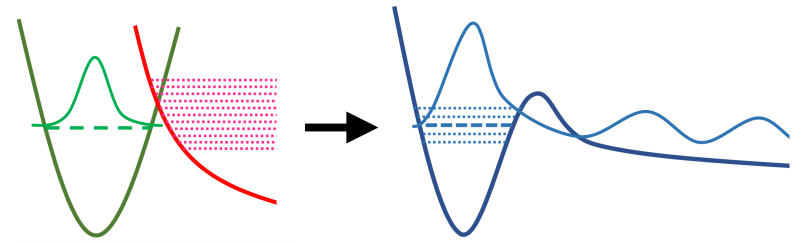

FIG. 2. In Feshbach-Fano framework, a resonance state is described in terms of the interaction between bound and continuum states. These zero-order states form a diabatic basis in which the solution of the full Schrödinger equation can be represented. Feshbach introduced projector operators $Q$ and $P$, which define the separation of the full function space into the the bound and continuum domains. The diabatic zero-order states are the solutions of the Schrödinger equation in these artificially decoupled subspaces.

Such a decomposition of the wave function is the essence of the Feshbach-Fano approach[27, 28] for treating autoionizing states (resonances). As originally formulated by Fano[27], and shown in Fig. 2, the autoionization can be described in terms of mixing between discrete and continuum diabatic electronic states, coupled by the off-diagonal (i.e., continuum-bound) matrix elements of the many-body Hamiltonian. Feshbach put this idea on a more rigorous mathematical basis by using projector operators and the partitioning approach[28], known in the quantum chemistry community as the Löwdin partitioning technique[29]. This approach is the basis of our theoretical framework. Feshbach projection operators $Q$ and $P$ divide the full function space into the bound and continuum domains. Similar to Fano's picture, in Feshbach's construction the resonance state is described as a bound state from the discrete subspace $Q$ coupled to the continuum subspace $P$. By using the partitioning technique[29, 30], the Schrödinger equation can be mapped into an eigenproblem in the $Q$-space with an effective Hamiltonian incorporating the $P$-space. Most often, one treats the bound part of the Hamiltonian and the respective eigenstates as zero-order states and includes the effect of the continuum at the first-order perturbation, as was done in the original Feshbach paper[28]. The critical aspect of Feshbach-Fano formalism is that the quality of the results depends strongly on the choice of the projector operators, which are not rigorously defined. This is the main stumbling block for quantitative applications of the Feshbach-Fano formalism to many-body autoionization problems. Among recent attempts to develop physically and theoretically justified projectors, the work of Martin and co-workers[31] and Kunitsa and Bravaya[32] are notable.

Fortunately, in the case of the Auger decay, one can easily separate the bound and continuum many-body configurations in the Fock space. This is possible because (i) the core-level states are Feshbach resonances, which can only decay by a two-electron process, and (ii) the core orbitals are well separated from the valence orbitals. Thus, Slater determinants, in which at least one core orbital is active, form the bound domain, which can only couple to the continuum by pure valence excited determinants. This is exploited in the core-valence separation (CVS) [33] scheme commonly used to adapt standard electronic structure methods for treating core-level states[34-43]. The CVS ansatz decouples core-excited and core-ionized states from the valence continua, essentially acting as the Feshbach $Q$ projector. In standard applications of the CVS scheme, the continuum is simply ignored and the core-level states are treated as bound states (in terms of perturbation theory, one can think of these CVS states as zero-order states). Here we extend the theory and include the effect of the continuum by explicitly constructing the decay states and evaluating the matrix elements between the bound and continuum many-body states. In this way, we obtain first-order corrections to the energies of the core-level states: the real part of the correction adjusts the position of the resonance and the imaginary part gives its width.

The construction of the many-body decay states poses greater difficulties for the theory than the construction of the initial states (which can be treated by CVS) because the decay states belong to the continuum (Feshbach $P$ subspace) and cannot be properly represented with $\mathcal{L}^{2}$-integrable functions used in electronic structure calculations. The inherent difficulty of treating unbound many-electron systems[44-47] is the reason why calculations of the Auger decay rates are still not routine, even for small molecules. The existing approaches can be divided into three categories: $(i)$ methods that do not consider the state of the emitted 
Auger electron, ( $i i)$ methods that treat the Auger electron implicitly, without the continuum functions, and ( iii) methods that describe the Auger electron explicitly with a true continuum orbital.

The first category includes electron population analysis [48], in which the relative Auger rates are computed from the densities of the valence molecular orbitals on the atom with the core hole. In a similar spirit, statistical approaches estimate Auger spectra from the distribution of final products of the decay and their decomposition in terms of the weights of electronic configurations $[49,50]$. These methods are useful for larger systems with high density of the final states.

The second category comprises methods that treat the many-electron continuum states implicitly, by means of $\mathcal{L}^{2}$-integrable wave functions. This is done in the Green's operator formalism [51], non-Hermitian theories such as complex absorbing potential approach [52], or in the Stieltjes imaging procedure [53, 54]. Stieltjes imaging entails calculations of bound-continuum couplings by using a discretized representation of the continuum spectrum by an $\mathcal{L}^{2}$-integrable basis set. This approach has been combined with algebraic diagrammatic construction (ADC) within the Fano ansatz to compute Auger rates in atoms and small molecules [55$57]$.

In the third category of methods, the continuum character of the Auger electron is treated explicitly. The wave function for the final state is represented by an antisymmetrized product of a function for the bound molecular ion and a continuum orbital for the outgoing electron. In the early days, the bound ion was treated at the self-consistent mean-field level [58, 59]. More recently, various flavors of configuration interaction (CI) methods have been employed to calculate the bound part of the multi-electron wave function [6063]. The continuum orbital for each decay channel can be computed using a single-center expansion method and performing numerical integration of the effective one-electron Schrödinger equation with proper scattering boundary conditions [64]. These approaches have been shown to yield accurate results for Auger spectra of small systems such as $\mathrm{Ne}$ and $\mathrm{H}_{2} \mathrm{O}[60,62]$. For molecules, a one-center approximation is commonly employed, where it is assumed that the continuum orbital has the same form as in an atom bearing the core hole and relevant two-electron integrals have contributions only from the orbitals centered on that atom $[59,65]$. This approach is employed, for example, in the XMOLECULE package for modeling ultrafast dynamics in strong fields [66].

Although quite a few methods and algorithms for calculations of Auger spectra have been reported so far, their scope of applicability remains limited and their predictive power depends on the underlying $a b$ initio method. For example, for atoms, elaborate calculations of the Auger widths can be carried out with the multi-configurational Dirac-Fock method implemented in the RATIP program [67]. However, the results depend strongly on the manual selection of configurations included in the subspace for the initial and final states. For molecules, the most advanced method today is the Fano-ADC-Stieltjes approach $[55,56]$. The drawbacks of this approach are that it requires large, nonstandard orbital basis sets and that it relies on somewhat arbitrary division of the electronic configurations into bound and continuum subspaces. Clearly, there is a need for more-universal computational tools for reliable treatment of Auger decay. Ideally, such new computational protocols should be cost-effective, easy to setup, and take advantage of the already available, highly accurate methods and algorithms of standard quantum chemistry.

Here, we propose a methodology to calculate Auger decay rates based on equation-of-motion coupled cluster (EOM-CC) theory [68-71]. We use EOM-CC to describe the bound part of the wave function in the initial and final states of the Auger decay and use continuum orbitals to represent the Auger electrons. The EOM-CC framework provides effective and robust tools for computing energies and properties of excited, ionized, and electron-attached states [68-71]. The flexibility of the EOM-CC single-reference ansatz allows one to tackle states of open-shell and multi-configurational character with high and controllable accuracy. EOM$\mathrm{CC}$ methods have been combined with complex absorbing potentials to study properties of metastable states $[47,72,73]$. To enable access to core-level states, EOM-CC methods have been combined with CVS, resulting in a highly effective CVS-EOM-CC scheme. The CVS-EOM-CCSD approach has been used to compute energies and properties of core-ionized and core-excited states, as well as X-ray non-linear properties such as RIXS[34-36, 38, 39, 42]. Here we extend the EOM-CC methodology to describe the autoionization properties of core-ionized and core-excited states.

We combine many-electronic states described by CVS-EOM-CCSD with a continuum orbital, which we approximate by a plane wave or a Coulomb wave. This allows us to avoid numerical integration in the calculations of mixed bound-continuum electron-repulsion integrals. The working equations for the calculations of the partial autoionization widths are expressed in terms of one- and two-body Dyson functions, contracted with the bound-continuum integrals. While Dyson orbitals have been utilized in the theory of one-photon photoionization [74-81], here we extend this concept to two-body functions, which enables a compact representation of autoionization widths obtained from correlated manyelectron states. In this paper, we describe the theoretical approach and its implementation in an electronic structure code. In a companion paper[82], we illustrate the performance of the theory by simulating normal and resonant Auger decay spectra in a set of benchmark 
atomic and molecular systems, including $\mathrm{Ne}, \mathrm{H}_{2} \mathrm{O}, \mathrm{CH}_{4}$, and $\mathrm{CO}_{2}$.

\section{FESHBACH-FANO-LÖWDIN FRAMEWORK}

As outlined above, our treatment of the autoionization process is based on the concepts originally formulated by Feshbach to describe nuclear reactions [28]. This is an application of the Löwdin partitioning technique[29] to treat the bound-continuum problem. While the focus of this paper is on the Auger effect, the theory is general and can be applied to other resonance phenomena[32].

Let us start by reviewing the key concepts of the approach. The principal idea $[28,29]$ is the introduction of two Hermitian, mutually orthogonal, projection operators $Q$ and $P$, such that:

$$
Q+P=1, \quad Q P=P Q=0 .
$$

The operators $Q$ and $P$ divide the full function space into two subspaces: the $Q$-space, characterizing the interaction region with the discrete spectrum, and the $P$-space, characterizing the asymptotic region with the continuous spectrum. The projection operators can be expressed as:

$$
Q=\sum_{n}\left|\psi_{n}\right\rangle\left\langle\psi_{n}\left|, \quad P=\sum_{\mu} \int_{0}^{\infty} d E\right| \chi_{\mu, E}^{ \pm}\right\rangle\left\langle\chi_{\mu, E}^{ \pm}\right|
$$

where the representing functions are the eigenstates of the respective projected Hamiltonians:

$$
H_{Q Q} \psi_{n}=E_{n} \psi_{n}, \quad H_{P P} \chi_{\mu, E}^{ \pm}=\left(E+E_{\mu}\right) \chi_{\mu, E}^{ \pm}
$$

with $H_{Q Q} \equiv Q H Q$ and $H_{P P} \equiv P H P$. These functions are subject to the following normalization conditions:

$$
\left\langle\psi_{n} \mid \psi_{k}\right\rangle=\delta_{n k}, \quad\left\langle\chi_{\mu, E}^{ \pm} \mid \chi_{\mu^{\prime}, E^{\prime}}^{ \pm}\right\rangle=\delta_{\mu \mu^{\prime}} \delta\left(E-E^{\prime}\right) .
$$

Thus, functions forming the $Q$-space are $\mathcal{L}^{2}$-normalized, whereas the $P$-space comprises scattering (unbound) states with Dirac's $\delta$ normalization. For the unbound states $\chi_{\mu, E}^{ \pm}$the index $\mu$ denotes a distinct open channel and the superscript \pm refers to either outgoing or incoming asymptotic boundary conditions imposed on the scattering wave function. In Eq. (3) we introduced $E_{\mu}$ which denotes the threshold energy of a given channel, i.e., $E_{\mu}$ corresponds to the internal energies of the two subsystems formed after the break-up. Similarly to the Fano picture[27], a resonance in the Feshbach theory can be seen as an isolated bound state from the $Q$-space, interacting with a bath of continuum states from the $P$ space. This interaction (or coupling) is responsible for the decay of the resonance. For this construction to be valid, the operator $P$ must include the summation over all possible open channels $\mu$ contributing to the decay of the given resonant state.

The $Q$ and $P$ operators transform the full Schrödinger equation

$$
H \Psi=E \Psi
$$

into an equivalent set of two sets of coupled equations, represented as

$$
\left[\begin{array}{ll}
H_{Q Q} & H_{Q P} \\
H_{P Q} & H_{P P}
\end{array}\right]\left[\begin{array}{l}
Q \Psi \\
P \Psi
\end{array}\right]=E\left[\begin{array}{l}
Q \Psi \\
P \Psi
\end{array}\right] .
$$

These two equations can be rearranged to define two effective Hamiltonians, $\mathcal{H}_{P P}$ and $\mathcal{H}_{Q Q}$ :

$$
\begin{gathered}
\mathcal{H}_{P P}=H_{P P}+H_{P Q} G_{Q}(E) H_{Q P} \\
\mathcal{H}_{Q Q}=H_{Q Q}+H_{Q P} G_{P}^{(+)}(E) H_{P Q}
\end{gathered}
$$

where $G_{Q}$ and $G_{P}^{(+)}$are the Green's functions in the $Q$ and $P$-spaces:

$$
\begin{array}{r}
G_{Q}(E)=\frac{1}{E-H_{Q Q}}, \\
G_{P}^{(+)}(E)=\lim _{\epsilon \rightarrow 0} \frac{1}{E+i \epsilon-H_{P P}} .
\end{array}
$$

Both $\mathcal{H}_{P P}$ and $\mathcal{H}_{Q Q}$ are energy-dependent and nonlocal[30]. They act only in their respective subspaces, yet, due to the presence of the coupling $H_{P Q / Q P}$, they include also the effect of the complementary subspace. Apparently equivalent, the $\mathcal{H}_{P P}$ and $\mathcal{H}_{Q Q}$ effective Hamiltonians have different properties and applications. $\mathcal{H}_{P P}$ is sufficient to obtain the asymptotic form of the total wave function, Eq. (5), and thus to calculate all scattering properties of the system.

By construction, the effective Hamiltonian $\mathcal{H}_{Q Q}$ is non-Hermitian and has complex eigenvalues. These eigenvalues are not equal to the eigenvalues of the original Hamiltonian, Eq. (5), solved with normal boundary conditions. Rather, they represent the solution of the Siegert-type problem[83] using modified boundary conditions, as in other incarnations of the non-Hermitian quantum mechanics[46] designed to describe resonance states by using $\mathcal{L}^{2}$ representation of the wave function.

Indeed, if $P$ and $Q$ operators are defined in an adequate way, then the eigenstates of $\mathcal{H}_{Q Q}$

$$
\mathcal{H}_{Q Q} \tilde{\psi}_{n}=\tilde{\mathcal{E}}_{n} \tilde{\psi}_{n}
$$

can be identified with true resonances and their respective eigenvalues

$$
\tilde{\mathcal{E}}_{n}=\mathcal{E}_{n}-i \frac{\Gamma_{n}}{2}
$$

correspond to physical observables, i.e., the position $\left(\mathcal{E}_{n}\right)$ and the width $\left(\Gamma_{n}\right)$ of the resonance. 
As in the context of electron correlation, the exact solution of $\mathcal{H}_{Q Q}$ is impractical. Instead, perturbation theory can employed, taking $H_{Q Q}$ as the zero-order Hamiltonian and treating the rest as a perturbation[30, 8486]. Thus, the eigenstates of $H_{Q Q}$ are zero-order wavefunctions:

$$
H_{Q Q} \psi_{n}=E_{n} \psi_{n}
$$

and $E_{n}$ is zero-oder energy of the resonance (because $H_{Q Q}$ is Hermitian, $E_{n}$ is real). The first-order correc- tion to the energy is then:

$$
E_{n}^{(1)}=\left\langle\psi_{n}\left|H_{Q P} G_{P}^{(+)} H_{P Q}\right| \psi_{n}\right\rangle .
$$

By using the distribution property:

$$
\lim _{\epsilon \rightarrow 0} \frac{1}{x \pm i \epsilon}=P . V \cdot \frac{1}{x} \mp i \pi \delta(x),
$$

we arrive at the following expressions for the resonance position:

$$
\mathcal{E}_{n}=\operatorname{Re}\left\langle\psi_{n}\left|\mathcal{H}_{Q Q}\right| \psi_{n}\right\rangle=E_{n}+\Delta_{n}=E_{n}+\sum_{\mu} P . V . \int_{0}^{\infty} d E \frac{\left\langle\psi_{n}\left|H_{Q P}\right| \chi_{\mu, E}^{ \pm}\right\rangle\left\langle\chi_{\mu, E}^{ \pm}\left|H_{P Q}\right| \psi_{n}\right\rangle}{E_{n}-E_{\mu}-E} \equiv E_{n}+\sum_{\mu} \Delta_{\mu, n},
$$

and for the resonance width

$$
\Gamma_{n}=-2 \operatorname{Im}\left\langle\psi_{n}\left|\mathcal{H}_{Q Q}\right| \psi_{n}\right\rangle=\sum_{\mu} 2 \pi\left\langle\psi_{n}\left|H_{Q P}\right| \chi_{\mu, E_{n}-E_{\mu}}^{ \pm}\right\rangle\left\langle\chi_{\mu, E_{n}-E_{\mu}}^{ \pm}\left|H_{P Q}\right| \psi_{n}\right\rangle \equiv \sum_{\mu} \Gamma_{\mu, n},
$$

in the first order of the perturbation theory. The second term in Eq. (16) represents shift to the position of the resonance due to the coupling with the continuum. Both the energy shift $\Delta_{n}$ and the width $\Gamma_{n}$ are sums over partial contributions from each open channel $\mu$.

We note that this treatment is meaningful only if $\psi_{n}$ provides a good approximation to the resonance wavefunction and the perturbation does not change its character. In other words, this treatment is justified for isolated, non-overlapping resonances. In the above expression, the coupling $H_{Q P / P Q}$ are the matrix elements of the Hamiltonian between the $Q$ - and $P$-spaces and $\psi_{n}$ and $\chi_{\mu, E_{n}-E_{\mu}}^{ \pm}$are eigenstates of the zero-order Hamiltonian comprising the uncoupled $H_{Q Q}$ and $H_{P P}$ blocks.

\section{AUGER TRANSITION AMPLITUDES AND ONE- AND TWO-BODY DYSON FUNCTIONS}

Let us now discuss how to generate bound and continuum zero-order electronic states within EOM-CC framework and how to effectively compute the transition amplitudes $\left\langle\psi_{n}\left|H_{Q P}\right| \chi_{\mu, E}\right\rangle=\left\langle\psi_{n}\left|H-E_{n}\right| \chi_{\mu, E}\right\rangle$ entering the expressions for the partial widths and energy shifts. Our derivation follows, to some extent, the work of Manne and Ågren [87], who derived general expressions for the Auger amplitudes from manyelectron wave functions, albeit adjusted to accommodate coupled-cluster theory and our assumption about the continuum orbital. We denote the initial (bound) state from the $Q$-space as

$$
\left|\psi_{n}\right\rangle=\left|{ }^{S, M_{S}} \Psi_{n}^{N}\right\rangle
$$

where $S$ and $M_{S}$ are spin quantum numbers, and the superscript $N$ is the number of electrons. The final (continuum) states of the $N$-electron system after the autoionization can be represented as:

$$
\left.\left.\left|\chi_{\mu, E}\right\rangle=\left.c_{\alpha} \hat{a}_{\mathbf{k}, \alpha}^{\dagger}\right|^{S^{\prime}, M_{S}-\frac{1}{2}} \Psi_{\mu}^{N-1}\right\rangle+\left.c_{\beta} \hat{a}_{\mathbf{k}, \beta}^{\dagger}\right|^{S^{\prime}, M_{S}+\frac{1}{2}} \Psi_{\mu}^{N-1}\right\rangle
$$

where $\left|S^{\prime}, M_{S}-\frac{1}{2} \Psi_{\mu}^{N-1}\right\rangle$ denotes a stable, $N-1$ electron core, and $\hat{a}_{\mathbf{k}, \sigma}^{\dagger}$ are creation operators of the free electron of energy $E=\frac{k^{2}}{2}$ and spin $\sigma$. If the initial resonant state has energy $E_{n}$, then the energy of the ejected electron fulfills the following condition (in atomic units)

$$
E_{n}=\frac{k^{2}}{2}+E_{\mu}
$$

where $E_{\mu}$ is the energy of the stable ion. Constants $c_{\alpha}$ and $c_{\beta}$ are determined by spin adaptation and are expressed in terms of the Clebsch-Gordan coefficients as

$c_{\alpha}=\left\langle\frac{1}{2}, \frac{1}{2} ; S^{\prime}, M_{S}-\frac{1}{2} \mid S, M_{S}\right\rangle, c_{\beta}=\left\langle\frac{1}{2},-\frac{1}{2} ; S^{\prime}, M_{S}+\frac{1}{2} \mid S, M_{S}\right\rangle$.

In this way the final continuum state $\chi$ has the same total spin $S$ as the initial state, which is the consequence of the spin conservation in the course of auto-ionization. From the angular momentum algebra, we know that possible spins of the final ion states are $S^{\prime}=S \pm \frac{1}{2}$. Without loss of generality, we can assume that the initial state has non-negative spin projection, i.e., $M_{S} \geq 0$, and in the following we consider the continuum state in the simplified form as

$$
\left|\chi_{\mu, E}\right\rangle=\hat{a}_{\mathbf{k}, \alpha}^{\dagger}\left|S^{\prime}, M_{S}-\frac{1}{2} \Psi_{\mu}^{N-1}\right\rangle
$$


where there is only one component with $\alpha$ spin of the free electron. To account for the properly spin-adapted form of $\chi$, Eq. (19), a degeneracy factor defined as:

$$
g_{\alpha}=\frac{1}{c_{\alpha}^{2}}
$$

is included in the final expressions for partial widths $\Gamma_{n, \mu}$ and energy shifts $\Delta_{n, \mu}$. Thus, from now on, we assume that the Auger electron has spin $\alpha$ and drop all spin quantum numbers in states labeling as they only enter the final expressions via the degeneracy factor $g_{\alpha}$.

In what follows we assume strong orthogonality condition - that is, that the continuum orbital corresponding to $\hat{a}_{\mathbf{k}}$ operator is orthogonal to all orbitals from the bound domain present in $\left|\Psi_{n}^{N}\right\rangle$ or $\left|\Psi_{\mu}^{N-1}\right\rangle$ states. This "killer condition" can be formally expressed as:

$$
\hat{a}_{\mathbf{k}}\left|\Psi_{n}^{N}\right\rangle=\hat{a}_{\mathbf{k}}\left|\Psi_{\mu}^{N-1}\right\rangle=0,\left\langle\Psi_{n}^{N}\right| \hat{a}_{\mathbf{k}}^{\dagger}=\left\langle\Psi_{\mu}^{N-1}\right| \hat{a}_{\mathbf{k}}^{\dagger}=0 .
$$

In the derivation of transition amplitudes, we express the Hamiltonian in the second quantization form:

$$
H=\hat{O}_{1}+\hat{O}_{2}=\oiint_{p q} h_{p q} \hat{a}_{p}^{\dagger} \hat{a}_{q}+\frac{1}{2} \oiint_{p q r s} g_{p q r s} \hat{a}_{p}^{\dagger} \hat{a}_{q}^{\dagger} \hat{a}_{s} \hat{a}_{r}
$$

where $h_{p q}$ denotes the one-electron integrals (kinetic energy and nuclear-electron interaction), $g_{p q r s}$ denotes electron-repulsion integrals $\langle p q \mid r s\rangle$, and the symbol $\&$ signifies that this summation includes spin-orbitals from both the bound and continuum domains. The creation and annihilation operators fulfill the anti-commutation relation:

$$
\hat{a}_{p}^{\dagger} \hat{a}_{q}+\hat{a}_{q} \hat{a}_{p}^{\dagger}=\delta_{p q}
$$

By employing strong orthogonality and using anticommutation properties, the one-electron part of the right transition amplitude assumes the following form

$$
\begin{aligned}
\left\langle\Psi_{n}^{N}\left|\hat{O}_{1}\right| \hat{a}_{\mathbf{k}}^{\dagger} \Psi_{\mu}^{N-1}\right\rangle & =\left\langle\Psi_{n}^{N}\left|\oiint_{p q} h_{p q} \hat{a}_{p}^{\dagger} \hat{a}_{q} \hat{a}_{\mathbf{k}}^{\dagger}\right| \Psi_{\mu}^{N-1}\right\rangle \\
& =\sum_{p} h_{p \mathbf{k}}^{n \mu} \gamma^{p}
\end{aligned}
$$

where we have introduced one-body (right) Dyson amplitudes ${ }^{n \mu} \gamma^{p}$ defined as

$$
{ }^{n \mu} \gamma^{p}=\left\langle\Psi_{n}^{N}\left|\hat{a}_{p}^{\dagger}\right| \Psi_{\mu}^{N-1}\right\rangle,
$$

which connect the $N$ and $N-1$ electron states. The last summation in Eq. (26) is now restricted to the spinorbitals from the bound domain only (no superimposed integral sign). ${ }^{n \mu} \gamma^{p}$ are the coefficients of Dyson orbital $\phi^{d}$ expressed in the molecular orbital basis set:

$$
\phi^{d}\left(\mathbf{x}_{1}\right)=\sum_{p}^{n \mu} \gamma^{p} \phi_{p}^{*}\left(\mathbf{x}_{1}\right)
$$

or, equivalently, as a generalized overlap integral in the first quantization:

$$
\begin{aligned}
\phi^{d}\left(\mathbf{x}_{1}\right)= & \sqrt{N} \int\left(\Psi_{n}^{N}\left(\mathbf{x}_{1}, \mathbf{x}_{2}, \ldots, \mathbf{x}_{N}\right)\right)^{*} \\
& \times \Psi_{\mu}^{N-1}\left(\mathbf{x}_{2}, \ldots, \mathbf{x}_{N}\right) d \mathbf{x}_{2} \ldots d \mathbf{x}_{N} .
\end{aligned}
$$

Likewise, the one-electron part of the left amplitude is

$$
\begin{aligned}
\left\langle\Psi_{\mu}^{N-1}\left|\hat{a}_{\mathbf{k}} \hat{O}_{1}\right| \Psi_{n}^{N}\right\rangle & =\left\langle\Psi_{\mu}^{N-1}\left|\hat{a}_{\mathbf{k}} \oiint_{p q} h_{p q} \hat{a}_{p}^{\dagger} \hat{a}_{q}\right| \Psi_{n}^{N}\right\rangle \\
& =\sum_{p} h_{\mathbf{k} p}{ }^{\mu n} \gamma_{p}
\end{aligned}
$$

where one-body (left) Dyson amplitudes ${ }^{\mu n} \gamma^{p}$

$$
{ }^{\mu n} \gamma_{p}=\left\langle\Psi_{\mu}^{N-1}\left|\hat{a}_{p}\right| \Psi_{n}^{N}\right\rangle
$$

have been introduced. Following the same procedure, the two-electron part of the right transition amplitude can be expressed as

$$
\begin{aligned}
\left\langle\Psi_{n}^{N}\left|\hat{O}_{2}\right| \hat{a}_{\mathbf{k}}^{\dagger} \Psi_{\mu}^{N-1}\right\rangle & =\left\langle\Psi_{n}^{N}\left|\frac{1}{2} \oint_{p q r s} g_{p q r s} \hat{a}_{p}^{\dagger} \hat{a}_{q}^{\dagger} \hat{a}_{s} \hat{a}_{r} \hat{a}_{\mathbf{k}}^{\dagger}\right| \Psi_{\mu}^{N-1}\right\rangle \\
& =\frac{1}{2} \sum_{p q r}\langle p q \| \mathbf{k} r\rangle^{n \mu} \Gamma_{r}^{p q}
\end{aligned}
$$

where we used the symmetrized two-electron integrals

$$
\langle p q \| \mathbf{k} r\rangle=g_{p q \mathbf{k} r}-g_{p q r \mathbf{k}}
$$

and two-body (right) Dyson amplitudes ${ }^{n \mu} \Gamma_{r}^{p q}$ defined as

$$
{ }^{n \mu} \Gamma_{r}^{p q}=\left\langle\Psi_{n}^{N}\left|\hat{a}_{p}^{\dagger} \hat{a}_{q}^{\dagger} \hat{a}_{r}\right| \Psi_{\mu}^{N-1}\right\rangle .
$$

Analogously to Dyson orbitals, ${ }^{n \mu} \Gamma_{r}^{p q}$ are the coefficients of the two-body Dyson function:

$$
g^{d}\left(\mathbf{x}_{1}, \mathbf{x}_{2}, \mathbf{x}_{2^{\prime}}\right)=\sum_{p q r}{ }^{R} \Gamma_{r}^{p q} \phi_{p}\left(\mathbf{x}_{\mathbf{1}}\right)^{*} \phi_{q}\left(\mathbf{x}_{\mathbf{2}}\right)^{*} \phi_{r}\left(\mathbf{x}_{\mathbf{2}^{\prime}}\right),
$$

which, again, can be equivalently written down in the first-quantization formalism as the following overlap integral:

$$
\begin{gathered}
g^{d}\left(\mathbf{x}_{1}, \mathbf{x}_{2}, \mathbf{x}_{2^{\prime}}\right)=\frac{\sqrt{N(N-1)}}{2} \int\left(\Psi_{n}^{N}\left(\mathbf{x}_{1}, \mathbf{x}_{2}, \ldots, \mathbf{x}_{N}\right)\right)^{*} \\
\times \Psi_{\mu}^{N-1}\left(\mathbf{x}_{2^{\prime}}, \mathbf{x}_{3}, \ldots, \mathbf{x}_{N}\right) d \mathbf{x}_{3} \ldots d \mathbf{x}_{N} .
\end{gathered}
$$

The left counterpart of the two-electron transition amplitude is

$$
\begin{aligned}
\left\langle\Psi_{\mu}^{N-1}\left|\hat{a}_{\mathbf{k}} \hat{O}_{2}\right| \Psi_{n}^{N}\right\rangle & =\left\langle\Psi_{\mu}^{N-1}\left|\hat{a}_{\mathbf{k}} \frac{1}{2} \oint_{p q r s} g_{p q r s} \hat{a}_{p}^{\dagger} \hat{a}_{q}^{\dagger} \hat{a}_{r} \hat{a}_{s}\right| \Psi_{n}^{N}\right\rangle \\
& =\frac{1}{2} \sum_{p q r}\langle\mathbf{k} r \| p q\rangle^{\mu n} \Gamma_{p q}^{r}
\end{aligned}
$$

where the (left) two-body Dyson amplitudes ${ }^{\mu n} \Gamma_{q r}^{p}$ are

$$
{ }^{\mu n} \Gamma_{q r}^{p}=\left\langle\Psi_{\mu}^{N-1}\left|\hat{a}_{p}^{\dagger} \hat{a}_{q} \hat{a}_{r}\right| \Psi_{n}^{N}\right\rangle .
$$


One- and two-body Dyson amplitudes, as defined by Eqs. (27) and (34), are analogous objects. The onebody Dyson function can be obtained by integrating the two-body Dyson function; respectively, the onebody Dyson amplitudes can be obtained by tracing the two-body amplitudes. Eqs. (29) and (36) also highlight the relationship between Dyson amplitudes and oneand two-body transition density matrices, commonly used objects in the electronic structure theory[81]. The difference between the transition density matrices and Dyson amplitudes is that the former connect the states with the same number of electrons, whereas the latter connect the states with a different number of electrons. Obviously, in the context of autoionization, one- and two-body Dyson functions are the key quantities, as they show how the initial resonance state is coupled with stable decay products. In the context of photoionization, the norms of one-body Dyson orbitals provide estimates of the strength of the transition (pole strengths) $[75,76,80,81]$, i.e., they are close to one for primary Koopmans-like transitions and are small for transitions with two-electron character (satellite transitions). In the same fashion, the norms of the twobody Dyson orbitals can be used to estimate relative Auger rates, in the spirit of electron population analysis approach[48] and density-matrix based estimates of electronic couplings [88, 89].

One- and two-body Dyson functions provide all the information about the resonance decay that can be distilled from $\mathcal{L}^{2}$-integrable wave functions. One- and twobody Dyson functions are bound-domain properties and can be calculated with electronic structure methods designed to tackle regular bound states. The remaining piece of the information about the resonance decay (from the unbound domain) is contained in the state of the emitted Auger electron, $\phi_{\mathbf{k}}$.

By combining all expressions for one- and twoelectron transition amplitudes and inserting them into Eq. (17) we arrive at the following formula for the resonance partial width:

$$
\Gamma_{n, \mu}=2 \pi g_{\alpha} \int d \Omega_{\mathbf{k}}\left(\sum_{p} h_{p \mathbf{k}}{ }^{n \mu} \gamma^{p}+\frac{1}{2} \sum_{p q r}\langle p q \| \mathbf{k} r\rangle^{n \mu} \Gamma_{r}^{p q}\right)\left(\sum_{p} h_{\mathbf{k} p}{ }^{\mu n} \gamma_{p}+\frac{1}{2} \sum_{p q r}\langle\mathbf{k} r \| p q\rangle^{\mu n} \Gamma_{p q}^{r}\right)
$$

and the correction for the resonance position:

$$
\Delta_{n, \mu}=g_{\alpha} P . V . \int d E \int d \Omega_{\mathbf{k}} \frac{\left(\sum_{p} h_{p \mathbf{k}}{ }^{n \mu} \gamma^{p}+\frac{1}{2} \sum_{p q r}\langle p q \| \mathbf{k} r\rangle^{n \mu} \Gamma_{r}^{p q}\right)\left(\sum_{p} h_{\mathbf{k} p}{ }^{\mu n} \gamma_{p}+\frac{1}{2} \sum_{p q r}\langle\mathbf{k} r \| p q\rangle^{\mu n} \Gamma_{p q}^{r}\right)}{E_{n}-E_{\mu}-E}
$$

where we have included also the degeneracy factor $g_{\alpha}$, and an explicit integration over the angles $\Omega_{\mathbf{k}}$ of the emitted electron with the momentum $\mathbf{k}$.

One and two-electron integrals, $h_{p \mathbf{k}}$ and $\langle p q \| \mathbf{k} r\rangle$, are mixed integrals between the orbitals from the bound domain and the continuum orbital $\phi_{\mathbf{k}}$ describing the emitted electron. Explicit expression for one-electron mixed integrals reads:

$$
h_{p \mathbf{k}}=\left\langle\phi_{p}\left|-\frac{1}{2} \nabla_{\mathbf{r}}^{2}+\sum_{i} \frac{Z_{i}}{\left|\mathbf{r}-\mathbf{A}_{i}\right|}-E_{n}\right| \phi_{\mathbf{k}}\right\rangle
$$

and for two-electron mixed integrals:

$$
\begin{aligned}
\langle p q \| \mathbf{k} r\rangle= & \left\langle\phi_{p}(1) \phi_{q}(2) \mid \frac{1}{\left|\mathbf{r}_{1}-\mathbf{r}_{2}\right|} \phi_{\mathbf{k}}(1) \phi_{r}(2)\right\rangle \\
& -\left\langle\phi_{p}(1) \phi_{q}(2) \mid \frac{1}{\left|\mathbf{r}_{1}-\mathbf{r}_{2}\right|} \phi_{r}(1) \phi_{\mathbf{k}}(2)\right\rangle .
\end{aligned}
$$

Importantly, the orbitals from the bound domain and the continuum orbital are subject to different normalization:

$$
\left\langle\phi_{p} \mid \phi_{q}\right\rangle=\delta_{p q}, \quad\left\langle\phi_{\mathbf{k}} \mid \phi_{\mathbf{k}^{\prime}}\right\rangle=\delta\left(E-E^{\prime}\right)
$$

in order to fulfill the normalization conditions imposed on the many-body electronic states, as defined in Eq. (4).

Eqs. (39) and (40) use left and right Dyson functions, which are not simple conjugates of each other in nonHermitian frameworks, such as CC/EOM-CC. In the case of Hermitian approaches, these equations reduce to contain the absolute squares of one amplitude.

\section{EOM-CCSD STATES FOR REGULAR AND RESONANT AUGER EFFECTS}

Let us now discuss how to employ EOM-CC methods to compute necessary electronic states and the corresponding one and two-body Dyson functions for Auger phenomena. Within the EOM-CC framework, the target state is parameterized as

$$
\left|\Psi_{I}\right\rangle=\hat{R}_{I} e^{\hat{T}}\left|\Phi_{0}\right\rangle
$$

where $\left|\Phi_{0}\right\rangle$ is a reference determinant, $\hat{T}$ is the excitation cluster operator from the CC ansatz, and $\hat{R}_{I}$ is a generalized EOM excitation operator. Different types 


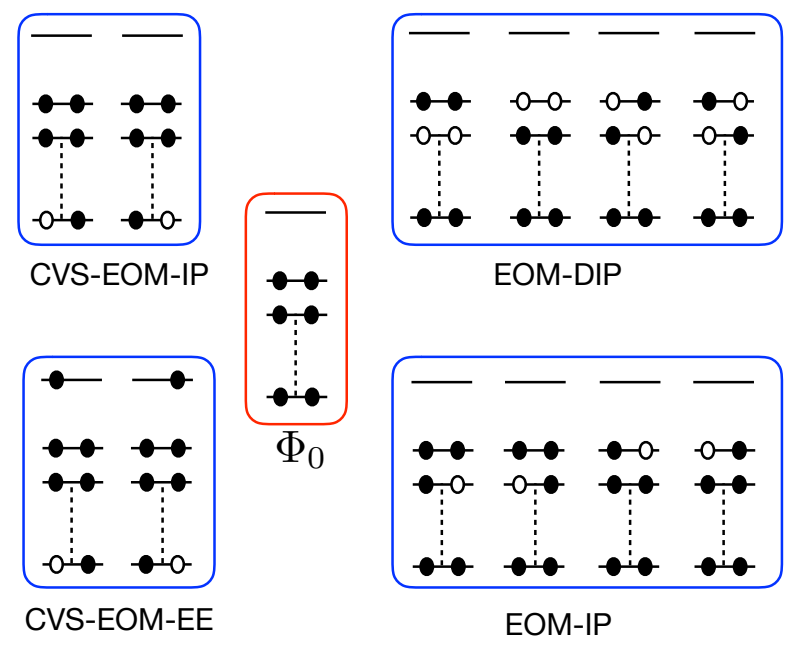

FIG. 3. Target spaces accessed by different EOM-CC models (only singly excited configurations are shown) from the closed-shell reference state $\left(\Phi_{0}\right)$.

of $\hat{R}$ (electron-conserving excitation, electron attaching, electron-removing, etc) allow access to different sectors of the Fock space[68], as illustrated in Fig. 3. Appropriate selection of $\hat{R}_{I}$ operator is a crucial step in the calculations, because $\hat{R}_{I}$ determines the initial resonance state $\left(\psi_{n}\right.$, Eq. (18)) and its possible decay channels.

Here we employ the CCSD ansatz (coupled-cluster with single and double excitations) in which the cluster operator $\hat{T}$ is restricted to single and double excitations:

$$
\hat{T}=\hat{T}_{1}+\hat{T}_{2}=\sum_{i a} t_{i}^{a} \hat{a}_{a}^{\dagger} \hat{a}_{i}+\frac{1}{4} \sum_{i j a b} t_{i j}^{a b} \hat{a}_{a}^{\dagger} \hat{a}_{b}^{\dagger} \hat{a}_{j} \hat{a}_{i} .
$$

Following standard notations, occupied and unoccupied spin-orbitals in $\left|\Phi_{0}\right\rangle$ are denoted by $i, j, k \ldots$ and $a, b, c \ldots$ indices, respectively. The level of excitation in the EOM-CC operators is chosen appropriately, e.g., $1 h 1 p$ and $2 h 2 p$ in EOM-EE, $1 h$ and $2 h 1 p$ in EOM-IP, $2 h$ and $3 h 1 p$ in EOM-DIP, and so on (here $h$ and $p$ denote hole and particle).

To compute transition properties within EOM-CC theory, we need also left EOM states, defined as:

$$
\left\langle\Psi_{I}\right|=\left\langle\Phi_{0}\right| \hat{L}_{I} e^{-\hat{T}}
$$

where $\hat{L}_{I}$ is a generalized EOM deexcitation operator.

The EOM-CC operators $R$ and $L$ are the eigenstates of the non-Hermitian similarity-transformed Hamiltonian $\bar{H}$ :

$$
\bar{H}=e^{-\hat{T}} H e^{\hat{T}} .
$$

Diagonalization of $\bar{H}$ in the space of target configurations, determined by a specific choice of $R$, yields EOM eigenvalues $E_{n}$, together with the corresponding left and right eigenvectors, satisfying the following equations:

$$
\bar{H} \hat{R}_{I}=E_{n} \hat{R}_{I}, \quad \hat{L}_{I} \bar{H}=E_{n} \hat{L}_{I} .
$$

Because of the non-Hermiticity of $\bar{H}$, the EOM-CC eigenvectors are not orthonormal in the usual sense, but are chosen to form a bioorthonormal set

$$
\left\langle\Psi_{I} \mid \Psi_{J}\right\rangle=\left\langle\Phi_{0} \mid \hat{L}_{I} \hat{R}_{J} \Phi_{0}\right\rangle=\delta_{I J} .
$$

The choice of the EOM operator $\hat{R}_{I}$ depends on the physical process we aim to describe. Different types of Auger processes are illustrated in Fig. 1. We assume that the Auger effect can be treated as a twostep process, with the first step (core-ionization or coreexcitation) being independent from the second step at which the Auger electron is emitted.

In regular Auger decay (Fig. 1a), which is relevant to XPS experiments, the initial state is a core-ionized state and target (decay) states are doubly ionized valence states. These states can be accessed by CVSEOM-IP and EOM-DIP, respectively, as illustrated in Fig. 3.

The CVS scheme restricts the target EOM-IP manifold to include only the configurations in which at least one core electron is active; in this way the coupling with the pseudo-continuum is removed and the core state becomes bound. This is achieved by splitting the occupied spin-orbitals into the core (denoted by capital indices $I, J \ldots)$ and valence (denoted by lower-case indices) sets. In our variant[36] of CVS-EOM-CC approach, we also use frozen-core approximation, such that the cluster operator in Eq. (45) is restricted to excitations from the valence shell only.

\section{The CVS-IP-CCSD states are}

$$
\left|\Psi_{n}^{N-1}\right\rangle=\hat{R}_{I P}^{C V S} e^{\hat{T}}\left|\Phi_{0}^{N}\right\rangle, \quad\left\langle\Psi_{n}^{N-1}\right|=\left\langle\Phi_{0}^{N}\right| \hat{L}_{I P}^{C V S} e^{-\hat{T}}
$$

where the right and left operators are

$$
\hat{R}_{I P}^{C V S}=\sum_{I} r_{I} \hat{a}_{I}+\frac{1}{2} \sum_{I J a} r_{I J}^{a} \hat{a}_{a}^{\dagger} \hat{a}_{J} \hat{a}_{I}+\sum_{I j a} r_{I j}^{a} \hat{a}_{a}^{\dagger} \hat{a}_{j} \hat{a}_{I}
$$

and

$$
\hat{L}_{I P}^{C V S}=\sum_{I} l^{I} \hat{a}_{I}^{\dagger}+\frac{1}{2} \sum_{I J a} l_{a}^{I J} \hat{a}_{I}^{\dagger} \hat{a}_{J}^{\dagger} \hat{a}_{a}+\sum_{I j a} l_{a}^{I j} \hat{a}_{I}^{\dagger} \hat{a}_{j}^{\dagger} \hat{a}_{a} .
$$

The products of the Auger decay correspond to doubly ionized states with two holes in the valence shell, described by EOM-DIP-CCSD:

$$
\left|\Psi_{\mu}^{N-2}\right\rangle=\hat{R}_{D I P} e^{\hat{T}}\left|\Phi_{0}^{N}\right\rangle, \quad\left\langle\Psi_{\mu}^{N-2}\right|=\left\langle\Phi_{0}^{N}\right| \hat{L}_{D I P} e^{-\hat{T}},
$$

where the right and left EOM operators are given by

$$
\hat{R}_{D I P}=\frac{1}{2} \sum_{i j} r_{i j} \hat{a}_{j} \hat{a}_{i}+\frac{1}{6} \sum_{i j k a} r_{i j k}^{a} \hat{a}_{a}^{\dagger} \hat{a}_{k} \hat{a}_{j} \hat{a}_{i}
$$


and

$$
\hat{L}_{D I P}=\frac{1}{2} \sum_{i j} l^{i j} \hat{a}_{i}^{\dagger} \hat{a}_{j}^{\dagger}+\frac{1}{6} \sum_{i j k a} l_{a}^{i j k} \hat{a}_{i}^{\dagger} \hat{a}_{j}^{\dagger} \hat{a}_{k}^{\dagger} \hat{a}_{a} .
$$

Here we can point out some major advantages of the EOM-CC approach. First, the EOM-CC ansatz naturally captures multi-configurational character of the initial and product states by treating leading electronic configurations on the same footing. When using closedshell references, the EOM-CC wave-functions are naturally spin-adapted[90]. Second, both the initial and final product states include dynamical correlation effects, described by higher-order excitation operators, cf. Eqs (51), (52), (54), (55). Third, both the initial and final product states are calculated by diagonalization of the same model Hamiltonian $\bar{H}$ and using the same set of orthogonal spin-orbitals from the bound domain, which significantly simplifies the formalism and leads to a balanced description of the states involved. Fourth, this consistent treatment of the resonance and its decay channels guarantees that we properly identify the open channels.

As explained above, the only properties needed from the bound-domain calculations are one- and two-body Dyson functions. With the initial state and final channel states defined by Eqs. (50) and (53) one-body Dyson functions vanish, which reflects the fact that the Auger decay is a two-electron process. Two-body Dyson functions for the regular Auger decay are given by the following expressions:

$$
\begin{aligned}
& { }^{n \mu} \Gamma_{r}^{p q}=\left\langle\Phi_{0}^{N}\left|\hat{L}_{I P}^{C V S} e^{-\hat{T}} \hat{a}_{p}^{\dagger} \hat{a}_{q}^{\dagger} \hat{a}_{r} \hat{R}_{D I P} e^{\hat{T}}\right| \Phi_{0}^{N}\right\rangle, \\
& { }^{\mu n} \Gamma_{q r}^{p}=\left\langle\Phi_{0}^{N}\left|\hat{L}_{D I P} e^{-\hat{T}} \hat{a}_{p}^{\dagger} \hat{a}_{q} \hat{a}_{r} \hat{R}_{I P}^{C V S} e^{\hat{T}}\right| \Phi_{0}^{N}\right\rangle .
\end{aligned}
$$

The programmable expressions for these matrix elements within the EOM-CCSD model are given in the Appendix.

The second example is the resonant Auger effect, relevant for XAS experiments. The difference from the regular Auger effect is that now the initial resonance state is created by core-valence excitation rather than by core ionization (see Fig. 1b,c). The initial states are, therefore, described by CVS-EOM-EE-CCSD (see Fig. 3 ), with left and right target states given by

$$
\left|\Psi_{n}^{N}\right\rangle=\hat{R}_{E E}^{C V S} e^{\hat{T}}\left|\Phi_{0}^{N}\right\rangle, \quad\left\langle\Psi_{n}^{N}\right|=\left\langle\Phi_{0}^{N}\right| \hat{L}_{E E}^{C V S} e^{-\hat{T}},
$$

with the CVS-EOM-EE-CCSD operators

$$
\begin{aligned}
\hat{R}_{E E}^{C V S}= & r_{0}+\sum_{I a} r_{I}^{a} \hat{a}_{a}^{\dagger} \hat{a}_{I}+\frac{1}{4} \sum_{I J a b} r_{I J}^{a b} \hat{a}_{a}^{\dagger} \hat{a}_{b}^{\dagger} \hat{a}_{J} \hat{a}_{I} \\
& +\frac{1}{2} \sum_{I j a b} r_{I j}^{a b} \hat{a}_{a}^{\dagger} \hat{a}_{b}^{\dagger} \hat{a}_{j} \hat{a}_{I} \\
\hat{L}_{E E}^{C V S}= & \sum_{I a} l_{a}^{I} \hat{a}_{I}^{\dagger} \hat{a}_{a}+\frac{1}{4} \sum_{I J a b} l_{a b}^{I J} \hat{a}_{I}^{\dagger} \hat{a}_{J}^{\dagger} \hat{a}_{b} \hat{a}_{a} \\
& +\frac{1}{2} \sum_{I j a b} l_{a b}^{I j} \hat{a}_{I}^{\dagger} \hat{a}_{j}^{\dagger} \hat{a}_{b} \hat{a}_{a} .
\end{aligned}
$$

The final states in this case are described by EOM-IPCCSD

$$
\left|\Psi_{\mu}^{N-1}\right\rangle=\hat{R}_{I P} e^{\hat{T}}\left|\Phi_{0}^{N}\right\rangle, \quad\left\langle\Psi_{\mu}^{N-1}\right|=\left\langle\Phi_{0}^{N}\right| \hat{L}_{I P} e^{-\hat{T}},
$$

where the right and left EOM-IP-CCSD operators are given by

$$
\begin{aligned}
\hat{R}_{I P} & =\sum_{i} r_{i} \hat{a}_{i}+\frac{1}{2} \sum_{i j a} r_{i j}^{a} \hat{a}_{a}^{\dagger} \hat{a}_{j} \hat{a}_{i}, \\
\hat{L}_{I P} & =\sum_{i} l^{i} \hat{a}_{i}^{\dagger}+\frac{1}{2} \sum_{i j a} l_{a}^{i j} \hat{a}_{i}^{\dagger} \hat{a}_{j}^{\dagger} \hat{a}_{a} .
\end{aligned}
$$

In resonant Auger effect, one distinguishes between participator and spectator decay. In the former, the electron originally excited from the core-shell takes part also in the decay process (Fig. 1b), whereas in the latter this electron remains in the excited orbital (Fig. 1c). The channels for the spectator decay require at least $2 h 1 p$ configurations, therefore, within the EOM-CCSD ansatz, they are described less accurately than the participator channels (requiring only $1 h$ configurations).

As in the case of the regular Auger effect, all what is needed to compute $\Gamma$ and $\Delta$ are one- and two-body Dyson functions. Because of the $r_{0}$ term in the $\hat{R}_{E E S}^{C V S}$ operator, this time there is a non-vanishing contribution to the left one-body Dyson function (see Appendix); the right one-body Dyson function is zero. The main contribution to the decay amplitude comes, again, from the two-body Dyson functions, represented as:

$$
\begin{aligned}
& { }^{n \mu} \Gamma_{r}^{p q}=\left\langle\Phi_{0}^{N}\left|\hat{L}_{E E}^{C V S} e^{-\hat{T}} \hat{a}_{p}^{\dagger} \hat{a}_{q}^{\dagger} \hat{a}_{r} \hat{R}_{I P} e^{\hat{T}}\right| \Phi_{0}^{N}\right\rangle, \\
& { }^{\mu n} \Gamma_{q r}^{p}=\left\langle\Phi_{0}^{N}\left|\hat{L}_{I P} e^{-\hat{T}} \hat{a}_{p}^{\dagger} \hat{a}_{q} \hat{a}_{r} \hat{R}_{E E}^{C V S} e^{\hat{T}}\right| \Phi_{0}^{N}\right\rangle .
\end{aligned}
$$

The programmable expressions for these Dyson functions in terms of the $\mathrm{CC}$ and $\mathrm{EOM}$ amplitudes are given in the Appendix.

\section{CONTINUUM ORBITAL AND MIXED BOUND-FREE MOLECULAR INTEGRALS}

Let us now discuss the issue of the continuum orbital $\phi_{\mathbf{k}}$, and evaluation of mixed bound-continuum one- and two-electron integrals. With the definition of the manybody continuum wave function as given in Eq. (21), 
the continuum orbital $\phi_{\mathbf{k}}$ describes the motion of the ejected electron in the field created by the residual ion $\left|\Psi_{\mu}\right\rangle . \phi_{\mathbf{k}}$ can be obtained by solving a Hartree-Fock like equation (rigorously derived from the Kohn variational method) $[87,91]$ :

$$
\begin{aligned}
& {\left[-\frac{1}{2} \nabla_{\mathbf{r}}^{2}-\sum_{A} \frac{Z_{A}}{\left|\mathbf{r}-\mathbf{R}_{\mathbf{A}}\right|}+\hat{J}\left[\Psi_{\mu}\right](\mathbf{r})-\hat{K}\left[\Psi_{\mu}\right](\mathbf{r})\right] \phi_{\mathbf{k}}(\mathbf{r})} \\
& =\frac{k^{2}}{2} \phi_{\mathbf{k}}(\mathbf{r}),
\end{aligned}
$$

subject to the strong orthogonality and normalization conditions as given by Eqs. $(23,43)$. In this equation, the Coulomb and the exchange operators are defined as

$$
\hat{J}\left[\Psi_{\mu}\right](\mathbf{r}) \phi_{\mathbf{k}}(\mathbf{r})=\int d \mathbf{r}^{\prime} \sum_{p q}^{\mu} \rho_{p q} \frac{\phi_{p}^{*}\left(\mathbf{r}^{\prime}\right) \phi_{q}\left(\mathbf{r}^{\prime}\right)}{\left|\mathbf{r}-\mathbf{r}^{\prime}\right|} \phi_{\mathbf{k}}(\mathbf{r})
$$

and

$$
\hat{K}\left[\Psi_{\mu}\right](\mathbf{r}) \phi_{\mathbf{k}}(\mathbf{r})=\int d \mathbf{r}^{\prime} \sum_{p q}^{\mu} \rho_{p q} \frac{\phi_{p}^{*}\left(\mathbf{r}^{\prime}\right) \phi_{\mathbf{k}}\left(\mathbf{r}^{\prime}\right)}{\left|\mathbf{r}-\mathbf{r}^{\prime}\right|} \phi_{q}(\mathbf{r}),
$$

respectively. Operators $\hat{J}$ and $\hat{K}$ depend on the state of the residual ion $\left|\Psi_{\mu}\right\rangle$ through the one-particle state density matrix:

$$
{ }^{\mu} \rho_{p q}=\left\langle\Psi_{\mu}\left|\hat{a}_{p}^{\dagger} \hat{a}_{q}\right| \Psi_{\mu}\right\rangle .
$$

State density matrix ${ }^{\mu} \rho_{p q}$ comprises two blocks $\left({ }^{\mu} \rho_{\alpha \alpha}\right.$ and ${ }^{\mu} \rho_{\beta \beta}$ ) depending on the spin functions of $p$ and $q$ spin-orbitals. The Coulomb operator $\hat{J}$ has contribution from both components, whereas the exchange operator $\hat{K}$ has contribution only from ${ }^{\mu} \rho_{\alpha \alpha}$ component (assuming that the ejected electron has $\alpha$ spin).

The most common approach to solve Eq. (64) is to apply partial wave decomposition to $\phi_{\mathbf{k}}$, and then to approximate the exchange potential $\hat{K}\left[\Psi_{\mu}\right](\mathbf{r})$ with some simple model such as from homogeneous electron gas $[60,64]$. As a result, one arrives with a set of coupled second-order differential equations, which are solved numerically. Although such procedure works well for small atoms, it becomes impractical for larger, nonsymmetric molecules, in which one needs to account for non-spherical potential and deal with a slow convergence of the partial wave expansion. In the present work we do not attempt to solve Eq. (64) explicitly. Rather, we assume a simple form of the continuum function $\phi_{\mathbf{k}}$, either as a plane wave or a Coulomb wave.

Our first model for continuum orbital $\phi_{\mathbf{k}}$ is a plane wave:

$$
\phi_{\mathbf{k}}^{P W}(\mathbf{r})=\sqrt{\frac{k}{(2 \pi)^{3}}} e^{i \mathbf{k} \cdot \mathbf{r}}
$$

where the prefactor $\sqrt{k /(2 \pi)^{3}}$ results from normalization condition, Eq. (43). Continuum orbital in the form of a plane wave corresponds to the solution of Eq. (64) where we neglect all the potential terms. Although this might seem as a drastic approximation, one can argue that in the case of the Auger effect, the energy of ejected electron is so large (hundreds of $\mathrm{eV}$ ) that the potential of the ionized core may turn out to be small relative to the kinetic energy. Validity of this argument is discussed in the companion paper[82], where we present numeric results illustrating different treatments of the Auger electron.

The major advantage of approximating $\phi_{\mathbf{k}}$ with a plane wave is that we can directly perform analytic evaluation of all mixed one- and two-electron integrals, Eqs. $(41,42)$, provided that the orbitals from the bound domain are expanded in terms of Gaussian functions of the form

$$
\phi^{G}(\mathbf{r})=\left(x-A_{x}\right)^{i}\left(y-A_{y}\right)^{j}\left(z-A_{z}\right)^{l} e^{-\alpha|\mathbf{r}-\mathbf{A}|^{2}},
$$

which is the usual case. In the analytic evaluation of mixed Gaussian/plane-wave integrals we make use of the following property,

$$
e^{-\alpha|\mathbf{r}-\mathbf{A}|^{2}} e^{i \mathbf{k} \cdot \mathbf{r}}=e^{i \mathbf{k} \cdot \mathbf{A}} e^{-\frac{k^{2}}{2 \alpha}} e^{-\frac{\alpha}{2}|\mathbf{r}-\mathbf{A}|^{2}} e^{-\frac{\alpha}{2}\left|\mathbf{r}-\mathbf{A}-i \frac{\mathbf{k}}{\alpha}\right|^{2}}
$$

which shows that the product of a Gaussian and a planewave functions can be expressed as a product of two Gaussians with one of them centered in the complex plane. In this way, we can mimic a plane wave with a single $s$-type Gaussian, shifted to the complex plane. Thus, in the evaluation of mixed integrals, we can reuse the integral codes designed for Gaussian functions, after some simple modifications, based on the Eq. (70). Indeed, directly from Eq. (70) one can see that the overlap between a Gaussian and a plane wave is equal to the overlap of two (modified) Gaussians. Mixed integral with the kinetic energy operator can be simply reduced to the overlap integral since

$$
\int d \mathbf{r} \phi^{G}(\mathbf{r})\left(-\frac{1}{2} \nabla_{\mathbf{r}}^{2}\right) e^{i \mathbf{k} \cdot \mathbf{r}}=\frac{1}{2} k^{2} \int d \mathbf{r} \phi^{G}(\mathbf{r}) e^{i \mathbf{k} \cdot \mathbf{r}} .
$$

For the evaluation of one and two-electron Coulomb mixed integrals with the plane wave, we can use again Eq. (70) and replace a plane wave with a single $s$ Gaussian. The only complication arising for Coulomb integrals with a plane wave is that now we need to compute Boys function for a complex argument, as a consequence of positioning one Gaussian in the complex plane. In our implementation we have used the algorithm from Ref. [92] to evaluate complex-valued Boys function.

In our second model we approximate $\phi_{\mathbf{k}}$ with a Coulomb wave of the form

$$
\begin{aligned}
\phi_{\mathbf{k}}^{C W}(\mathbf{r})= & \sqrt{\frac{k}{(2 \pi)^{3}}} e^{i \mathbf{k} \cdot \mathbf{r}} \Gamma(1-i \eta) e^{-\frac{\pi \eta}{2}} \\
& \times{ }_{1} F_{1}(i \eta, 1,-i k r-i \mathbf{k} \cdot \mathbf{r})
\end{aligned}
$$


where $\eta=-Z / k$ is the Sommerfeld parameter, $Z$ is the nuclear charge, ${ }_{1} F_{1}$ is the Kummer confluent hypergeometric function, and the incoming wave boundary conditions are implied. This form of $\phi_{\mathbf{k}}$ corresponds to the assumption that the potential part from Eq. (64) can be approximated as $V(\mathbf{r})=-\frac{Z}{r}$, where $Z$ is an effective Coulomb charge $\left(Z_{\text {eff }}\right)$; its optimal value is discussed in the accompanying paper[82]. For $Z=0$ the Coulomb wave reduces to the plane wave.

As with the plane wave, we aim to evaluate mixed bound-continuum integrals analytically, without numerical integration. To do so, we employ the approach from Ref. [93], which provides a recipe for an efficient decomposition of the Coulomb wave in terms of products of Gaussian and plane-wave functions:

$$
\phi_{\mathbf{k}}^{C W}(\mathbf{r})=\sum c_{\mathbf{k}} \phi_{\mathbf{k}}^{G P W}(\mathbf{r}),
$$

where $\phi_{\mathbf{k}}^{G P W}(\mathbf{r})$ basis functions are in the form

$\phi_{\mathbf{k}}^{G P W}(\mathbf{r})=\left(x-A_{x}\right)^{i}\left(y-A_{y}\right)^{j}\left(z-A_{z}\right)^{l} e^{-\alpha|\mathbf{r}-\mathbf{A}|^{2}} e^{i \mathbf{k} \cdot(\mathbf{r}-\mathbf{A})}$.

The main idea behind the method of Ref. [93] is to rewrite the Coulomb wave as

$$
\phi_{\mathbf{k}}^{C W}(\mathbf{r})=\sqrt{\frac{k}{(2 \pi)^{3}}} e^{i \mathbf{k} \cdot \mathbf{r}} \sum_{l=0}^{\infty} \sum_{m=-l}^{l} \mathcal{O}_{k l}(r) \mathcal{R}_{l m}^{*}(\mathbf{r}) \mathcal{R}_{l m}(\mathbf{k}),
$$

where $\mathcal{O}_{k l}(r)$ are the $l$-th pseudo-partial waves given by

$\mathcal{O}_{k l}(r)=\Gamma(1-i \eta) e^{-\frac{\pi \eta}{2}}(i \eta)_{l} \frac{(-2 i)^{l}}{(2 l) !}{ }_{1} F_{1}(l+i \eta, 2 l+2,-2 i k r)$

and $\mathcal{R}_{l m}(\mathbf{v})$ are solid spherical harmonics of vector $\mathbf{v}$. The pseudo-partial waves $\mathcal{O}_{k l}(r)$ are functions that can be easily approximated with a small set of primitive Gaussians,

$$
\mathcal{O}_{k l}(r) \approx \sum_{i=i}^{N_{c}} c_{l_{i}} e^{-\xi_{l_{i}} r^{2}}
$$

where the exponents $\xi_{l_{i}}$ and expansion coefficients $c_{l_{i}}$ are determined via optimization procedure (done separately for each value of $k$ ). The advantages of this approach over the standard partial-wave expansion are two-fold. First, the major oscillatory part of the Coulomb wave is contained already in the $e^{i \mathbf{k} \cdot \mathbf{r}}$ term, making $\mathcal{O}_{k l}(r)$ smooth and slowly varying functions. Consequently, the expansion from Eq. (77) is very compact, even for very high energy (as we encounter in the Auger effect). Second, pseudo-partial wave convergence is substantially faster than for standard partial waves, again, owing to the explicit presence of $e^{i \mathbf{k} \cdot \mathbf{r}}$ factor in Eq. (75). Inserting $\mathcal{O}_{k l}(r)$ from Eq. (77) into definition from Eq. (75) leads directly to the expansion of the Coulomb wave in terms of $\phi_{\mathbf{k}}^{G P W}(\mathbf{r})$ functions. The next step is to evaluate the necessary mixed boundfree integrals with $\phi_{\mathbf{k}}^{G P W}(\mathbf{r})$ as a continuum orbital and Gaussian functions as remaining orbitals from the bound domain. For $\phi_{\mathbf{k}}^{G P W}(\mathbf{r})$ functions we make use of the following identity

$$
e^{-\alpha|\mathbf{r}-\mathbf{A}|^{2}} e^{i \mathbf{k} \cdot(\mathbf{r}-\mathbf{A})}=e^{-\frac{k^{2}}{4 \alpha}} e^{-\alpha\left|\mathbf{r}-\mathbf{A}-i \frac{\mathbf{k}}{2 \alpha}\right|^{2}},
$$

which shows that the $s$-type $\phi_{\mathbf{k}}^{G P W}(\mathbf{r})$ is equivalent to a regular $s$-type Gaussian, however, shifted to the complex plane. If $\phi_{\mathbf{k}}^{G P W}(\mathbf{r})$ is purely of $s$-type, the property above is sufficient to evaluate all mixed overlap and one and two-electron Coulomb integrals using standard integral codes for Gaussian functions (as for the plane wave, we need complex-valued Boys function for Coulomb integrals). If $\phi_{\mathbf{k}}^{G P W}(\mathbf{r})$ has non-zero angular momentum (in any direction), then mixed overlap and Coulomb integrals can be obtained from the horizontal recurrence relation, which allows to shift the angular momentum from one orbital centered on $\mathbf{A}$ to another orbital centered on B. Standard horizontal recurrence for one-electron integral $\left(i_{A} \mid i_{B}\right)$ can be schematically written as (assuming the shift is done along the $X$ axis):

$$
\left(i_{A} \mid i_{B}+1\right)=\left(i_{A}+1 \mid i_{B}\right)+\left(A_{x}-B_{x}\right) \cdot\left(i_{A} \mid i_{B}\right) .
$$

For mixed $\left(\phi^{G} \mid \phi^{G P W}\right)$ integrals this horizontal recurrence needs to be modified to

$$
\left(i_{A} \mid i_{B}+1\right)=\left(i_{A}+1 \mid i_{B}\right)+\operatorname{Re}\left(A_{x}-B_{x}\right) \cdot\left(i_{A} \mid i_{B}\right),
$$

so while $s$-type $\phi^{G P W}$ function is positioned in the complex plane (according to Eq. (78)), the horizontal shift to build angular momentum in $\phi^{G P W}$ is done only along the real axis. Separate treatment is needed for mixed kinetic energy integrals with $\phi^{G P W}$ function. For these integrals we can use the following property:

$$
\begin{aligned}
\int d \mathbf{r} \phi^{G}(\mathbf{r}) & \left(-\frac{1}{2} \nabla_{\mathbf{r}}^{2}\right) \phi_{\mathbf{k}}^{G P W}(\mathbf{r})= \\
& \int d \mathbf{r} \phi_{\mathbf{k}}^{G P W *}(\mathbf{r})\left(-\frac{1}{2} \nabla_{\mathbf{r}}^{2}\right) \phi^{G}(\mathbf{r}),
\end{aligned}
$$

and then by analyzing the action of differentiation operator onto the regular Gaussian function:

$$
\begin{aligned}
& \frac{\partial^{2}}{\partial x^{2}} \phi^{G}(\mathbf{r})= \\
& {\left[i(i-1) \frac{1}{\left(x-A_{x}\right)^{2}}+4 \alpha^{2}\left(x-A_{x}\right)^{2}-\alpha(4 i+2)\right] \phi^{G}(\mathbf{r}),}
\end{aligned}
$$

we show that the mixed kinetic energy integral can be simply related to the sum of overlap integrals.

Thus, by approximating the continuum orbital with either a plane or a Coulomb wave, we are able to evaluate all necessary mixed bound-continuum integrals by reusing standard integral codes for pure Gaussian functions (with some simple modifications). Therefore, all these integrals can be effectively computed using highly optimized codes developed for Gaussian integrals. Consequently, we will be able to apply our methodology 
to larger molecules, with sizable one-electron basis set, without additional cost due to numerical integration.

In our derivations, we assumed strong orthogonality of the continuum orbital, and it is clear that a plane wave or a Coulomb wave do not fulfill that condition without additional orthogonalization. However, as it was shown in Ref. [94], this orthogonalization is not necessary if the initial and final states are obtained from the same set of orthogonal bound-domain orbitals in a variational-like procedure (which is true for EOM-CC states). Therefore, we do not impose additional orthogonalization on the continuum orbital in our calculations.

\section{IMPLEMENTATION}

We implemented the calculation of one- and two-body Dyson amplitudes and mixed Gaussian-plane wave integrals in the developer's version of the Q-Chem quantum chemistry package[95, 96]. Our implementation used libtensor library[97] and the suite of CVS-EOM-EE codes recently developed by Coriani and co-workers[36].

All mixed Gaussian/plane-waves integrals were implemented using the libqint infrastructure. The twoelectron Coulomb integrals were computed by modifying the Head-Gordon-Pople algorithm [98] for electronrepulsion integrals and utilizing the implementation of White et al. [92] to calculate Boys function for a complex argument. Numerical integration over the angles of the emitted electron (Eq. 39) was done with Lebedev quadrature. Additional computational details are given in the companion paper[82].

\section{CONCLUSIONS}

We have presented the extension of the EOM-CCSD formalism to compute Auger decay rates in atoms and molecules. This work is a natural extension of previous developments based on the EOM-CCSD framework combined with the CVS scheme, and concerned with description of core-ionized and core-excited states. In the context of modeling autoionization, the advantages of the EOM-CC methods are: $(i)$ balanced description of the initial and final bound-domain wave functions with one set of orthogonal orbitals and the same effective Hamiltonian, (ii) simple, black-box computational setup, with no system-dependent parameterization, and (iii) flexibility to treat states of different electronic character, including multi-configurational and openshell wave-functions. To calculate Auger decay rates using the Feshbach-Fano ansatz, we have combined manyelectron CVS-EOM-IP/EE-CCSD states with a continuum orbital describing the outgoing electron, approximated by either a plane wave or a Coulomb wave.

In our companion paper[82], we present numeric examples, which illustrate the performance of the theory and highlight the consequence of approximate treatment of the free electron.

We conclude by noting that our methodology to calculate Auger widths is quite general and can be adapted to other problems concerned with autoionization, such as iteratomic Coulombic decay, electron-transfer mediated decay, or Penning ionization. Also, the theory can be particularly useful to generate smooth complex potential energy surfaces to study coupled electronic and nuclear dynamics in the presence of autoionization.

\section{ACKNOWLEDGMENTS}

This work was supported by the U.S. National Science Foundation (No. CHE-1856342). We thank Dr. Evgeny Epifanovsky from Q-Chem, Inc for his help and guidance in the implementation of mixed gaussian-plane wave integrals.

\section{CONFLICTS OF INTEREST} Inc.

A.I.K. is the president and a part-owner of Q-Chem,

\section{DATA AVAILABILITY STATEMENT}

The data that supports the findings of this study are available within the article and its supplementary material.

\section{APPENDIX}

Below we present EOM-CCSD programmable expressions for different blocks of one- $\left({ }^{n \mu} \gamma^{p} \equiv{ }^{R} \gamma^{p},{ }^{\mu n} \gamma^{p} \equiv{ }^{L} \gamma^{p}\right)$ and two-body $\left({ }^{n \mu} \Gamma_{r}^{p q} \equiv{ }^{R} \Gamma_{r}^{p q},{ }^{\mu n} \Gamma_{q r}^{p} \equiv{ }^{L} \Gamma_{q r}^{p}\right)$ Dyson amplitudes for the relevant combinations of the EOM models. Two-body Dyson functions have the following permutational symmetry:

$$
{ }^{R} \Gamma_{r}^{p q}=-{ }^{R} \Gamma_{r}^{q p} \text { and }{ }^{L} \Gamma_{q r}^{p}=-{ }^{L} \Gamma_{r q}^{p} .
$$

In the following, we make use of the symmetrizing/anti-symmetrizing operator $\mathcal{P}^{ \pm}(i, j)$ defined as:

$$
\mathcal{P}^{ \pm}(i, j)[f(i, j)]=f(i, j) \pm f(j, i),
$$


and adapt notation for right and left one- and two-body Dyson amplitudes:

$$
{ }^{n \mu} \gamma^{p} \equiv{ }^{R} \gamma^{p},{ }^{\mu n} \gamma^{p} \equiv{ }^{L} \gamma^{p},{ }^{n \mu} \Gamma_{r}^{p q} \equiv{ }^{R} \Gamma_{r}^{p q},{ }^{\mu n} \Gamma_{q r}^{p} \equiv{ }^{L} \Gamma_{q r}^{p} .
$$

CVS-EOM-IP-CCSD to EOM-DIP-CCSD Dyson amplitudes

$$
\begin{gathered}
R \gamma^{p}=\left\langle\Phi_{0}\left|\hat{L}_{I P}^{C V S} e^{-\hat{T}} \hat{a}_{p}^{\dagger} e^{\hat{T}} \hat{R}_{D I P}\right| \Phi_{0}\right\rangle=0 \\
{ }^{R} \Gamma_{J}^{a i}=\left\langle\Phi_{0}\left|\hat{L}_{I P}^{C V S} e^{-\hat{T}} \hat{a}_{a}^{\dagger} \hat{a}_{i}^{\dagger} \hat{a}_{J} e^{\hat{T}} \hat{R}_{D I P}\right| \Phi_{0}\right\rangle=-\sum_{k} l_{a}^{J k} r_{i k} \\
{ }^{R} \Gamma_{K}^{i j}=\left\langle\Phi_{0}\left|\hat{L}_{I P}^{C V S} e^{-\hat{T}} \hat{a}_{i}^{\dagger} \hat{a}_{j}^{\dagger} \hat{a}_{K} e^{\hat{T}} \hat{R}_{D I P}\right| \Phi_{0}\right\rangle=\sum_{l a} l_{a}^{K l} r_{i j l}^{a}+l^{K} r_{i j}+\sum_{l a} l_{a}^{K l} t_{i}^{a} r_{j l}-\sum_{l a} l_{a}^{K l} t_{j}^{a} r_{i l} .
\end{gathered}
$$

EOM-DIP-CCSD to CVS-EOM-IP-CCSD Dyson amplitudes

$$
\begin{gathered}
{ }^{L} \gamma_{p}=\left\langle\Phi_{0}\left|\hat{L}_{D I P} e^{-\hat{T}} \hat{a}_{p} e^{\hat{T}} \hat{R}_{I P}^{C V S}\right| \Phi_{0}\right\rangle=0 \\
{ }^{L} \Gamma_{j k}^{I}=\left\langle\Phi_{0}\left|\hat{L}_{D I P} e^{-\hat{T}} \hat{a}_{I}^{\dagger} \hat{a}_{j} \hat{a}_{k} e^{\hat{T}} \hat{R}_{I P}^{C V S}\right| \Phi_{0}\right\rangle=-\sum_{l a} l_{a}^{j k l} r_{I l}^{a}-l^{j k} r_{I}, \\
{ }^{L} \Gamma_{a j}^{I}=\left\langle\Phi_{0}\left|\hat{L}_{D I P} e^{-\hat{T}} \hat{a}_{I}^{\dagger} \hat{a}_{a} \hat{a}_{j} e^{\hat{T}} \hat{R}_{I P}^{C V S}\right| \Phi_{0}\right\rangle=\sum_{k} l^{j k} r_{I k}^{a}+\sum_{k l b}\left(l_{b}^{j k l} \cdot r_{I l}^{b}\right) t_{k}^{a} \\
+r_{I} \sum_{k} t_{k}^{a} l^{j k}+\frac{1}{2} r_{I} \sum_{k l b} l_{b}^{j k l} t_{k l}^{a b}, \\
{ }^{L} \Gamma_{a b}^{I}=\left\langle\Phi_{0}\left|\hat{L}_{D I P} e^{-\hat{T}} \hat{a}_{I}^{\dagger} \hat{a}_{a} \hat{a}_{b} e^{\hat{T}} \hat{R}_{I P}^{C V S}\right| \Phi_{0}\right\rangle \\
=-\frac{1}{2} r_{I} \sum_{j k} l^{j k} t_{j k}^{a b}+\sum_{j k} t_{j}^{a} t_{k}^{b} \Gamma_{j k}^{I}-\frac{1}{2} \sum_{j k l c} l_{c}^{j k l} r_{I j}^{c} t_{k l}^{a b} \\
+\mathcal{P}^{-}(a, b)\left[\frac{1}{2} \sum_{j k l c}\left(l_{c}^{j k l} \cdot t_{k l}^{a c}\right)\left(r_{I j}^{b}+r_{I} t_{j}^{b}\right)+\sum_{j k}\left(l^{j k} \cdot t_{j}^{b}\right) r_{I k}^{a}\right]
\end{gathered}
$$

\section{CVS-EOM-EE-CCSD to EOM-IP-CCSD Dyson amplitudes}

$$
\begin{gathered}
R \gamma^{p}=\left\langle\Phi_{0}\left|\hat{L}_{E E}^{C V S} e^{-\hat{T}} \hat{a}_{p}^{\dagger} e^{\hat{T}} \hat{R}_{I P}\right| \Phi_{0}\right\rangle=0 \\
{ }^{R} \Gamma_{I}^{a b}=\left\langle\Phi_{0}\left|\hat{L}_{E E}^{C V S} e^{-\hat{T}} \hat{a}_{a}^{\dagger} \hat{a}_{b}^{\dagger} \hat{a}_{I} e^{\hat{T}} \hat{R}_{I P}\right| \Phi_{0}\right\rangle=-\sum_{j} l_{a b}^{I j} r_{j} . \\
{ }^{R} \Gamma_{J}^{a i}=\left\langle\Phi_{0}\left|\hat{L}_{E E}^{C V S} e^{-\hat{T}} \hat{a}_{a}^{\dagger} \hat{a}_{i}^{\dagger} \hat{a}_{J} e^{\hat{T}} \hat{R}_{I P}\right| \Phi_{0}\right\rangle=-l_{a}^{J} r_{i}-\sum_{k b} l_{a b}^{J k} r_{i k}^{b}-\sum_{b} t_{i}^{b} \Gamma_{J}^{a b}
\end{gathered}
$$




$$
\begin{aligned}
{ }^{R} \Gamma_{K}^{i j}= & \left\langle\Phi_{0}\left|\hat{L}_{E E}^{C V S} e^{-\hat{T}} \hat{a}_{i}^{\dagger} \hat{a}_{j}^{\dagger} \hat{a}_{K} e^{\hat{T}} \hat{R}_{I P}\right| \Phi_{0}\right\rangle=-\sum_{a} l_{a}^{K} r_{i j}^{a}-\frac{1}{2} \sum_{l a b}\left(l_{a b}^{K l} \cdot r_{l}\right) \cdot t_{i j}^{a b}+\sum_{a b} t_{j}^{b} t_{i}^{a R} \Gamma_{K}^{a b} \\
& +\mathcal{P}^{-}(i, j)\left[\frac{1}{2} r_{j} \sum_{l a b} l_{a b}^{K l} t_{i l}^{a b}-r_{i} \sum_{a} l_{a}^{K} t_{j}^{a}-\sum_{l a b} t_{j}^{a} \cdot\left(l_{a b}^{K l} \cdot r_{i l}^{b}\right)\right] .
\end{aligned}
$$

EOM-IP-CCSD to CVS-EE-CCSD Dyson amplitudes

$$
\begin{aligned}
& { }^{L} \gamma_{i}=\left\langle\Phi_{0}\left|\hat{L}_{I P} e^{-\hat{T}} \hat{a}_{i} e^{\hat{T}} \hat{R}_{E E}^{C V S}\right| \Phi_{0}\right\rangle=l^{i} r_{0}, \\
& { }^{L} \gamma_{I}=\left\langle\Phi_{0}\left|\hat{L}_{I P} e^{-\hat{T}} \hat{a}_{I} e^{\hat{T}} \hat{R}_{E E}^{C V S}\right| \Phi_{0}\right\rangle=0, \\
& { }^{L} \gamma_{a}=\left\langle\Phi_{0}\left|\hat{L}_{I P} e^{-\hat{T}} \hat{a}_{a} e^{\hat{T}} \hat{R}_{E E}^{C V S}\right| \Phi_{0}\right\rangle=\sum_{i} t_{i}^{a}{ }^{L} \gamma^{i}+\frac{1}{2} r_{0} \sum_{i j b} l_{b}^{i j} t_{i j}^{a b} . \\
& { }^{L} \Gamma_{i j}^{a}=\left\langle\Phi_{0}\left|\hat{L}_{I P} e^{-\hat{T}} \hat{a}_{a}^{\dagger} \hat{a}_{i} \hat{a}_{j} e^{\hat{T}} \hat{R}_{E E}^{C V S}\right| \Phi_{0}\right\rangle=-l_{a}^{i j} r_{0} . \\
& { }^{L} \Gamma_{j k}^{i}=\left\langle\Phi_{0}\left|\hat{L}_{I P} e^{-\hat{T}} \hat{a}_{i}^{\dagger} \hat{a}_{j} \hat{a}_{k} e^{\hat{T}} \hat{R}_{E E}^{C V S}\right| \Phi_{0}\right\rangle={ }^{L} \tilde{\Gamma}_{j k}^{i}+\mathcal{P}^{-}(j, k)\left[\delta_{i j}{ }^{L} \gamma_{k}\right] \\
& { }^{L} \tilde{\Gamma}_{j k}^{i}=\sum_{a} l_{a}^{j k} t_{i}^{a} r_{0} \\
& { }^{L} \Gamma_{j k}^{I}=\left\langle\Phi_{0}\left|\hat{L}_{I P} e^{-\hat{T}} \hat{a}_{I}^{\dagger} \hat{a}_{j} \hat{a}_{k} e^{\hat{T}} \hat{R}_{E E}^{C V S}\right| \Phi_{0}\right\rangle=\sum_{a} l_{a}^{j k} r_{I}^{a} \\
& { }^{L} \Gamma_{J k}^{I}=\left\langle\Phi_{0}\left|\hat{L}_{I P} e^{-\hat{T}} \hat{a}_{I}^{\dagger} \hat{a}_{J} \hat{a}_{k} e^{\hat{T}} \hat{R}_{E E}^{C V S}\right| \Phi_{0}\right\rangle=\delta_{I J}{ }^{L} \gamma_{k} \\
& { }^{L} \Gamma_{j K}^{I}=\left\langle\Phi_{0}\left|\hat{L}_{I P} e^{-\hat{T}} \hat{a}_{I}^{\dagger} \hat{a}_{j} \hat{a}_{K} e^{\hat{T}} \hat{R}_{E E}^{C V S}\right| \Phi_{0}\right\rangle=-\delta_{I K}{ }^{L} \gamma_{j} \\
& { }^{L} \Gamma_{i b}^{a}=\left\langle\Phi_{0}\left|\hat{L}_{I P} e^{-\hat{T}} \hat{a}_{a}^{\dagger} \hat{a}_{i} \hat{a}_{b} e^{\hat{T}} \hat{R}_{E E}^{C V S}\right| \Phi_{0}\right\rangle=-\sum_{j} l_{a}^{i j} t_{j}^{b} r_{0} \\
& { }^{L} \Gamma_{b c}^{a}=\left\langle\Phi_{0}\left|\hat{L}_{I P} e^{-\hat{T}} \hat{a}_{a}^{\dagger} \hat{a}_{b} \hat{a}_{c} e^{\hat{T}} \hat{R}_{E E}^{C V S}\right| \Phi_{0}\right\rangle=r_{0} \sum_{i j} l_{a}^{i j}\left(-\frac{1}{2} t_{i j}^{b c}-t_{i}^{b} t_{j}^{c}\right) \\
& { }^{L} \Gamma_{a j}^{i}=\left\langle\Phi_{0}\left|\hat{L}_{I P} e^{-\hat{T}} \hat{a}_{i}^{\dagger} \hat{a}_{a} \hat{a}_{j} e^{\hat{T}} \hat{R}_{E E}^{C V S}\right| \Phi_{0}\right\rangle={ }^{L} \tilde{\Gamma}_{a j}^{i}+\delta_{i j}\left({ }^{L} \gamma_{a}\right) \\
& { }^{L} \tilde{\Gamma}_{a j}^{i}=r_{0} l^{j} t_{i}^{a}+r_{0} \sum_{k b} l_{b}^{j k}\left(t_{i k}^{a b}-t_{i}^{b} t_{k}^{a}\right) \\
& { }^{L} \Gamma_{a j}^{I}=\left\langle\Phi_{0}\left|\hat{L}_{I P} e^{-\hat{T}} \hat{a}_{I}^{\dagger} \hat{a}_{a} \hat{a}_{j} e^{\hat{T}} \hat{R}_{E E}^{C V S}\right| \Phi_{0}\right\rangle=l^{j} r_{I}^{a}+\sum_{k b} l_{b}^{j k}\left(r_{I k}^{a b}-t_{k}^{a} r_{I}^{b}\right) \\
& { }^{L} \Gamma_{a J}^{I}=\left\langle\Phi_{0}\left|\hat{L}_{I P} e^{-\hat{T}} \hat{a}_{I}^{\dagger} \hat{a}_{a} \hat{a}_{J} e^{\hat{T}} \hat{R}_{E E}^{C V S}\right| \Phi_{0}\right\rangle=\delta_{I J}\left({ }^{L}{ }^{L} \gamma_{a}\right)
\end{aligned}
$$




$$
\begin{gathered}
{ }^{L} \Gamma_{a b}^{i}=\left\langle\Phi_{0}\left|\hat{L}_{I P} e^{-\hat{T}} \hat{a}_{i}^{\dagger} \hat{a}_{a} \hat{a}_{b} e^{\hat{T}} \hat{R}_{E E}^{C V S}\right| \Phi_{0}\right\rangle \\
=r_{0} \sum_{j} l^{j} t_{i j}^{a b}+\frac{1}{2} r_{0} \sum_{j k c}\left(l_{c}^{j k} \cdot t_{j k}^{a b}\right) \cdot t_{i}^{c}+r_{0} \sum_{j k} t_{j}^{a} t_{k}^{b}{ }^{L} \Gamma_{j k}^{i} \\
+r_{0} \mathcal{P}^{-}(a, b)\left[-\sum_{j k c}\left(l_{c}^{j k} \cdot t_{i j}^{a c}\right) \cdot t_{k}^{b}-\frac{1}{2} t_{i}^{b} \sum_{j k c} l_{c}^{j k} t_{j k}^{a c}\right] . \\
{ }^{L} \Gamma_{a b}^{I}=\left\langle\Phi_{0}\left|\hat{L}_{I P} e^{-\hat{T}} \hat{a}_{I}^{\dagger} \hat{a}_{a} \hat{a}_{b} e^{\hat{T}} \hat{R}_{E E}^{C V S}\right| \Phi_{0}\right\rangle \\
=\sum_{j} l^{j} r_{I j}^{a b}+\frac{1}{2} \sum_{j k c}\left(l_{c}^{j k} \cdot t_{j k}^{a b}\right) \cdot r_{I}^{c}+\sum_{j k} t_{j}^{a} t_{k}^{b} \Gamma_{j k}^{I} \\
+\mathcal{P}^{-}(a, b)\left[-r_{I}^{b} \sum_{j} t_{j}^{a} l^{j}-\sum_{j k c}\left(l_{c}^{j k} \cdot t_{j}^{a}\right) \cdot r_{I k}^{b c}-\frac{1}{2} r_{I}^{b} \sum_{j k c} l_{c}^{j k} t_{j k}^{a c}\right] .
\end{gathered}
$$


[1] B. K. Agarwal, X-ray spectroscopy: an introduction, volume 15. Springer, 2013.

[2] D. Spanjaard, C. Guillot, M.-C. Desjonqueres, G. Tréglia, and J. Lecante, Surface core level spectroscopy of transition metals: A new tool for the determination of their surface structure, Surf. Sci. Rep. 5, 1 (1985).

[3] M. Tchaplyguine, R. Feifel, R. R. T. Marinho, M. Gisselbrecht, S. L. Sorensen, A. N. de Brito, N. Mårtensson, S. Svensson, and O. Björneholm, Selective probing of the electronic structure of free clusters using resonant core-level spectroscopy, Chem. Phys. 289, 3 (2003).

[4] P. M. Kraus, M. Zürch, S. K. Cushing, D. M. Neumark, and S. R. Leone, The ultrafast x-ray spectroscopic revolution in chemical dynamics, Nat. Rev. Chem. 2, 82 (2018).

[5] B. K. McFarland, J. P. Farrell, S. Miyabe, F. Tarantelli, A. Aguilar, N. Berrah, C. Bostedt, J. D. Bozek, P. H. Bucksbaum, J. C. Castagna, R. N. Coffee, J. P. Cryan, L. Fang, R. Feifel, K. J. Gaffney, J. M. Glownia, T. J. Martinez, M. Mucke, B. Murphy, A. Natan, T. Osipov, V. S. Petrovi, S. Schorb, Th. Schultz, L. S. Spector, M. Swiggers, I. Tenney, S. Wang, J. L. White, W. White, and M. Gühr, Ultrafast x-ray Auger probing of photoexcited molecular dynamics, Nat. Comm. 5, 1 (2014).

[6] E. Gagnon, P. Ranitovic, X.-M. Tong, C. L. Cocke, M. M. Murnane, H. C. Kapteyn, and A. S. Sandhu, Soft $\mathrm{x}$-ray-driven femtosecond molecular dynamics, Science 317, 1374 (2007).

[7] M. Maiuri, M. Garavelli, and G. Cerullo, Ultrafast spectroscopy: state of the art and open challenges, J. Am. Chem. Soc. 142, 3 (2019).

[8] K. Ramasesha, S. R. Leone, and D. M. Neumark, Realtime probing of electron dynamics using attosecond time-resolved spectroscopy, Annu. Rev. Phys. Chem. 67, 41 (2016).

[9] H. Fukuzawa, T. Takanashi, E. Kukk, K. Motomura, S. Wada, K. Nagaya, Y. Ito, T. Nishiyama, Ch. Nicolas, Y. Kumagai, D. Iablonskyi, S. Mondal, T. Tachibana, D. You, S. Yamada, Y. Sakakibara, K. Asa, Y. Sato, T. Sakai, K. Matsunami, T. Umemoto, K. Kariyazono, S. Kajimoto, H. Sotome, P. Johnsson, M. S. Schöffler, G. Kastirke, K. Kooser, X.-J. Liu, T. Asavei, L. Neagu, S. Molodtsov, K. Ochiai, M. Kanno, K. Yamazaki, S. Owada, K. Ogawa, T. Katayama, T. Togashi, K. Tono, M. Yabashi, A. Ghosh, K. Gokhberg, L.S. Cederbaum, A.I. Kuleff, H. Fukumura, N. Kishimoto, A. Rudenko, K. Miron, H. Kono, and K. Ueda, Real-time observation of X-ray-induced intramolecular and interatomic electronic decay in $\mathrm{CH}_{2} \mathrm{I}_{2}$, Nat. Comm. 10, 1 (2019).

[10] Z.-H. Loh, G. Doumy, C. Arnold, L. Kjellsson, S. H. Southworth, A. Al Haddad, Y. Kumagai, M.-F. Tu, P. J. Ho, A. M. March, R. D. Schaller, M. S. Bin Mohd Yusof, T. Debnath, M. Simon, R. Welsch, L. Inhester, K. Khalili, K. D. Nanda, A. I. Krylov, S. Moeller, G. Coslovich, J. Koralek, M. P. Minitti, W. F. Schlotter, J.-E. Rubensson, R. Santra, and L. Young, Obser- vation of the fastest chemical processes in the radiolysis of water, Science 367, 179 (2020).

[11] L. Kjellsson, K. D. Nanda, J.-E. Rubensson, G. Doumy, S. H. Southworth, P. J. Ho, A. M. March, A. Al Haddad, Y. Kumagai, M.-F. Tu, T. Debnath, M. S. Bin Mohd Yusof, C. Arnold, W. F. Schlotter, S. Moeller, G. Coslovich, J. D. Koralek, M. P. Minitti, M. L. Vidal, M. Simon, R. Santra, Z.-H. Loh, S. Coriani, A. I. Krylov, and L. Young, Resonant inelastic x-ray scattering reveals hidden local transitions of the aqueous $\mathrm{OH}$ radical, Phys. Rev. Lett. 124, 236001 (2020).

[12] A. R. Attar, A. Bhattacherjee, C. D. Pemmaraju, K. Schnorr, K. D. Closser, D. Prendergast, and S. R. Leone, Femtosecond x-ray spectroscopy of an electrocyclic ring-opening reaction, Science 356, 54 (2017).

[13] H. J. Wörner, C. A. Arrell, N. Banerji, A. Cannizzo, M. Chergui, A. K. Das, P. Hamm, U. Keller, P. M. Kraus, E. Liberatore, P. Lopez-Tarifa, M. Lucchini, M. Meuwly, C. Milne, J.-E. Moser, U. Rothlisberger, G. Smolentsev, . Teuscher, J. A. van Bokhoven, and O. Wenger, Charge migration and charge transfer in molecular systems, Struct. Dyn. 4, 061508 (2017).

[14] T. Katayama, T. Northey, W. Gawelda, C. J. Milne, G. Vankó, F. A. Lima, Z. Németh, S. Nozawa, T. Sato, D. Khakhulin, J. Szlachetko, T. Togashi, S. Owada, S. Adachi, C. Bressler, M. Yabashi, and T. J. Penfold, Tracking multiple components of a nuclear wavepacket in photoexcited $\mathrm{Cu}(\mathrm{I})$-phenanthroline complex using ultrafast x-ray spectroscopy, Nat. Comm. 10, 1 (2019).

[15] H. Yong, N. Zotev, J. M. Ruddock, B. Stankus, M. Simmermacher, A. M. Carrascosa, W. Du, N. Goff, Y. Chang, D. Bellshaw, M. Liang, S. Carbajo, J. E. Koglin, J. S. Robinson, S. Boutet, M. P. Minitti, A. Kirrander, and P. M. Weber, Observation of the molecular response to light upon photoexcitation, Nat. Comm. 11, 1 (2020).

[16] M. Epshtein, V. Scutelnic, Z. Yang, T. Xue, M. L. Vidal, Anna I. Krylov, S. Coriani, and S. R. Leone, Table-top X-ray spectroscopy of benzene radical cation, J. Phys. Chem. A (2020), submitted; https://doi.org/10.26434/chemrxiv.12798734.v1.

[17] M. L. Vidal, M. Epshtein, V. Scutelnic, Z. Yang, T. Xue, S. R. Leone, A. I. Krylov, and S. Coriani, The interplay of open-shell spin-coupling and Jahn-Teller distortion in benzene radical cation probed by x-ray spectroscopy, J. Phys. Chem. A (2020), submitted; https://doi.org/10.26434/chemrxiv.12799922.v1.

[18] T. Åberg, Theory of the radiative Auger effect, Phys. Rev. A 4, 1735 (1971).

[19] R. Neutze, R. Wouts, D. Van der Spoel, E. Weckert, and J. Hajdu, Potential for biomolecular imaging with femtosecond x-ray pulses, Nature 406, 752 (2000).

[20] S. P. Hau-Riege, R. A. London, and A. Szoke, Dynamics of biological molecules irradiated by short x-ray pulses, Phys. Rev. E 69, 051906 (2004).

[21] C. C. Chang, Auger electron spectroscopy, Surf. Sci. 25, 53 (1971).

[22] S. Tougaard, Surface nanostructure determination by $\mathrm{x}$-ray photoemission spectroscopy peak shape analysis, 
J. Vac. Sci. Technol. A: Vacuum, Surfaces, and Films 14, 1415 (1996).

[23] C. J. Powell, A. Jablonski, I. S. Tilinin, S. Tanuma, and D. R. Penn, Surface sensitivity of Auger-electron spectroscopy and x-ray photoelectron spectroscopy, J. Electron. Spectrosc. Relat. Phenom. 98, 1 (1999).

[24] R. R. Rye, T. E. Madey, J. E. Houston, and P. H. Holloway, Chemical-state effects in Auger electron spectroscopy, J. Chem. Phys. 69, 1504 (1978).

[25] G. Wentzel, über strahlungslose Quantensprünge, Z. Physik 43, 521 (1927).

[26] T. Åberg and G. Howat, Theory of Auger effect, in Encyclopedia of Physics, edited by S. Flügge, volume 31. Berlin: Springer, 1982.

[27] U. Fano, Configuration interaction on intensities and phase shifts, Phys. Rev. 124, 1866 (1961).

[28] H. Feshbach, A unified theory of nuclear reactions. 2, Ann. Phys. (N.Y.) 19, 287 (1962).

[29] P.-O. Löwdin, Studies in perturbation theory. IV. Solution of eigenvalue problem by projection operator formalism, J. Math. Phys. 3, 969 (1962).

[30] D. A. Micha, Effective hamiltonian methods for molecular collisions, Adv. Quant. Chem. 8, 231 (1974).

[31] J. C. Cardona, J. L. Sanz-Vicario, and F. Martín, Complete Feshbach-type calculations of energy positions and widths of autoionizing states in Li-like atoms, Phys. Rev. A 82, 022501 (2010).

[32] A. A. Kunitsa and K. B. Bravaya, Feshbach projection XMCQDPT2 model for metastable electronic states, arXiv (2019), arXiv:1906.11390.

[33] L. S. Cederbaum, W. Domcke, and J. Schirmer, Manybody theory of core holes, Phys. Rev. A 22, 206 (1980).

[34] S. Coriani and H. Koch, Communication: X-ray absorption spectra and core-ionization potentials within a core-valence separated coupled cluster framework, J. Chem. Phys. 143, 181103 (2015).

[35] S. Coriani and H. Koch, Erratum: "Communication: X-ray absorption spectra and core-ionization potentials within a core-valence separated coupled cluster framework" [J. Chem. Phys. 143, 181103 (2015)], J. Chem. Phys. 145, 149901 (2016).

[36] M. L. Vidal, X. Feng, E. Epifanovski, A. I. Krylov, and S. Coriani, A new and efficient equation-of-motion coupled-cluster framework for core-excited and coreionized states, J. Chem. Theory Comput. 15, 3117 (2019).

[37] M. L. Vidal, A. I. Krylov, and S. Coriani, Dyson orbitals within the fc-CVS-EOM-CCSD framework: theory and application to X-ray photoelectron spectroscopy of ground and excited states, Phys. Chem. Chem. Phys. 22, 2693 (2020).

[38] R. Faber and S. Coriani, Resonant inelastic X-ray scattering and nonesonant X-ray emission spectra from coupled-cluster (damped) response theory, J. Chem. Theory Comput. 15, 520 (2019).

[39] K. D. Nanda, M. L. Vidal, R. Faber, S. Coriani, and A. I. Krylov, How to stay out of trouble in RIXS calculations within the equation-of-motion coupled-cluster damped response theory framework? Safe hitchhiking in the excitation manifold by means of core-valence separation, Phys. Chem. Chem. Phys. 22, 2629 (2020).
[40] R. Faber and S. Coriani, Core-valence-separated coupled-cluster-singles-and-doubles complexpolarization-propagator approach to X-ray spectroscopies, Phys. Chem. Chem. Phys. 22, 2642 (2020).

[41] K. D. Nanda and A. I. Krylov, A simple molecular orbital picture of RIXS distilled from many-body damped response theory, J. Chem. Phys. 152, 244118 (2020).

[42] K. D. Nanda and A. I. Krylov, Cherry-picking resolvents: A general strategy for convergent coupled-cluster damped response calculations of core-level spectra, J. Phys. Chem. 153, 141104 (2020).

[43] M. L. Vidal, P. Pokhilko, A. I. Krylov, and S. Coriani, Equation-of-motion coupled-cluster theory to model ledge x-ray absorption and photoelectron spectra, J. Phys. Chem. Lett. (2020), in press.

[44] W. P. Reinhardt, Complex coordinates in the theory of atomic and molecular structure and dynamics, Annu. Rev. Phys. Chem. 33, 223 (1982).

[45] N. Moiseyev, Quantum theory of resonances: Calculating energies, widths and cross-sections by complex scaling, Phys. Rep. 302, 212 (1998).

[46] N. Moiseyev, Non-Hermitian quantum mechanics. Cambridge University Press, 2011.

[47] T.-C. Jagau, K. B. Bravaya, and A. I. Krylov, Extending quantum chemistry of bound states to electronic resonances, Annu. Rev. Phys. Chem. 68, 525 (2017).

[48] M. Mitani, , O. Takahashi, K. Saito, and S. Iwata, Theoretical molecular Auger spectra with electron population analysis, J. Electron. Spectrosc. Relat. Phenom. 128, 103 (2003).

[49] F. Tarantelli, A. Sgamellotti, and L. S. Cederbaum, The calculation of molecular Auger spectra, J. Electron. Spectrosc. Relat. Phenom. 68, 297 (1994).

[50] T. R. Walsh, T. E. Meehan, and F. P. Larkins, Prediction of molecular Auger rates using a statistical model, J. Phys. B 27, 2211 (1994).

[51] B. Schimmelpfennig, B.M. Nestmann, and S.D. Peyerimhoff, Ab initio calculation of transition rates for autoionization: the auger spectra of $\mathrm{hf}$ and $\mathrm{f}^{-}$, J. Electron. Spectrosc. Relat. Phenom. 74, 173 (1995).

[52] A. Ghosh, N. Vaval, and S. Pal, Auger decay rates of core hole states using equation of motion coupled cluster method, Chem. Phys. 482, 160 (2017).

[53] C.-M. Liegener, Green's function calculations on the Auger spectra of CO, Chem. Phys. Lett. 106, 201 (1984).

[54] V. Carravetta and H. Ågren, Stieltjes imaging method for molecular Auger transition rates: Application to the Auger spectrum of water, Phys. Rev. A 35, 1022 (1987).

[55] P. Kolorenč and V. Averbukh, K-shell Auger lifetime variation in doubly ionized $\mathrm{Ne}$ and first row hydrides, J. Chem. Phys. 135, 134314 (2011).

[56] P. Kolorenč and V. Averbukh, Fano-ADC $(2,2)$ method for electronic decay rates, J. Chem. Phys. 152, 214107 (2020).

[57] V. Stumpf, S. Scheit, P. Kolorenč, and K. Gokhberg, Electron transfer mediated decay in nexe triggered by $K-L L$ auger decay of ne, Chem. Phys. 482, 192 (2017).

[58] G. Howat, T. Aberg, and O. Goscinski, Relaxation and final-state channel mixing in the Auger effect, J. Phys. B 11, 1575 (1978). 
[59] H. Siegbahn, L. Asplund, and P. Kelfve, The Auger electron spectrum of water vapour, Chem. Phys. Lett. 35, 330 (1975).

[60] L. Inhester, C. F. Burmeister, G. Groenhof, and H. Grubmueller, Auger spectrum of a water molecule after single and double core ionization, J. Chem. Phys. 136, 144304 (2012).

[61] S. Stock, R. Beerwerth, and S. Fritzsche, Auger cascades in resonantly excited neon, Phys. Rev. A 95, 053407 (2017).

[62] G. Grell, O. Kühn, and S. I. Bokarev, Multireference quantum chemistry protocol for simulating autoionization spectra: Test of ionization continuum models for the neon atom, Phys. Rev. A 100, 042512 (2019).

[63] G. Grell and S. I. Bokarev, Multi-reference protocol for (auto) ionization spectra: Application to molecules, J. Chem. Phys. 152, 074108 (2020).

[64] Ph. V. Demekhin, A. Ehresmann, and V. L. Sukhorukov, Single center method: A computational tool for ionization and electronic excitation studies of molecules, J. Chem. Phys. 134, 024113 (2011).

[65] F. P. Larkins, L. C. Tulea, and E. Z. Chelkowska, Auger electron spectra of molecules: the first row hydrides, Australian J. Phys. 43, 625 (1990).

[66] Y. Hao, L. Inhester, K. Hanasaki, S.-K. Son, and R. Santra, Efficient electronic structure calculation for molecular ionization dynamics at high x-ray intensity, Struct. Dyn. 2, 041707 (2015).

[67] S. Fritzsche, The RATIP program for relativistic calculations of atomic transition, ionization and recombination properties, Comp. Phys. Comm. 183, 1525 (2012).

[68] A. I. Krylov, Equation-of-motion coupled-cluster methods for open-shell and electronically excited species: The hitchhiker's guide to Fock space, Annu. Rev. Phys. Chem. 59, 433 (2008).

[69] J. F. Stanton and R. J. Bartlett, The equation of motion coupled-cluster method. A systematic biorthogonal approach to molecular excitation energies, transition probabilities, and excited state properties, J. Chem. Phys. 98, 7029 (1993).

[70] R. J. Bartlett, Coupled-cluster theory and its equationof-motion extensions, WIREs: Comput. Mol. Sci. 2, 126 (2012).

[71] K. Sneskov and O. Christiansen, Excited state coupled cluster methods, WIREs: Comput. Mol. Sci. 2, 566 (2012).

[72] T.-C. Jagau, D. Zuev, K. B. Bravaya, E. Epifanovsky, and A. I. Krylov, A fresh look at resonances and complex absorbing potentials: Density matrix based approach, J. Phys. Chem. Lett. 5, 310 (2014).

[73] D. Zuev, T.-C. Jagau, K. B. Bravaya, E. Epifanovsky, Y. Shao, E. Sundstrom, M. Head-Gordon, and A. I. Krylov, Complex absorbing potentials within EOMCC family of methods: Theory, implementation, and benchmarks, J. Chem. Phys. 141, 024102 (2014).

[74] O. Goscinski and P. Lindner, Natural spin-orbitals and generalized overlap amplitudes, J. Math. Phys. 11, 1313 (1970).

[75] J. Linderberg and Y. Öhrn, Propagators in quantum chemistry. Academic, London, 1973.

[76] J. V. Ortiz, Toward an exact one-electron picture of chemical bonding, Adv. Quantum Chem. 35, 33 (1999).
[77] H. R. Hudock, B. G. Levine, A. L. Thompson, H. Satzger, D. Townsend, N. Gador, S. Ulrich, A. Stolow, and T. J. Martínez, Ab initio molecular dynamics and time-resolved photoelectron spectroscopy of electronically excited uracil and thymine, J. Phys. Chem. A 111, 8500 (2007).

[78] C. M. Oana and A. I. Krylov, Dyson orbitals for ionization from the ground and electronically excited states within equation-of-motion coupled-cluster formalism: Theory, implementation, and examples, J. Chem. Phys. 127, 234106 (2007).

[79] S. Gozem, A. O. Gunina, T. Ichino, D. L. Osborn, J. F. Stanton, and A. I. Krylov, Photoelectron wave function in photoionization: Plane wave or Coulomb wave?, J. Phys. Chem. Lett. 6, 4532 (2015).

[80] J. V. Ortiz, Dyson-orbital concepts for description of electrons in molecules, J. Chem. Phys. 153, 070902 (2020).

[81] A. I. Krylov, From orbitals to observables and back, J. Chem. Phys. 153, 080901 (2020).

[82] W. Skomorowski and A. I. Krylov, Feshbach-fano approach for calculation of Auger decay rates using equation-of-motion coupled-cluster wave functions: $\mathrm{Nu}$ merical examples and benchmarks, J. Chem. Phys. (2020), ChemRxiv xxxxxxx.

[83] A. J. F. Siegert, On the derivation of dispersion formula for nuclear reactions, Phys. Rev. 56, 750 (1939).

[84] A. D. Bochevarov and C. D. Sherrill, Some simple results following from Löwdin's partitioning technique, J. Math. Chem. 42, 59 (2007).

[85] P.-O. Löwdin, Partitioning technique, perturbation theory, and rational approximations, Int. J. Quant. Chem. XXI, 69 (1982).

[86] C. Buth, R. Santra, and L. S. Cederbaum, NonHermitian Rayleigh-Schrödinger perturbation theory, Phys. Rev. A 69, 032505 (2004).

[87] R. Manne and H. Ågren, Auger transition amplitudes from general many-electron wavefunctions wave, Chem. Phys. 93, 201 (1985).

[88] X. Feng, A. V. Luzanov, and A. I. Krylov, Fission of entangled spins: An electronic structure perspective, J. Phys. Chem. Lett. 4, 3845 (2013).

[89] S. Matsika, X. Feng, A. V. Luzanov, and A. I. Krylov, What we can learn from the norms of one-particle density matrices, and what we can't: Some results for interstate properties in model singlet fission systems, J. Phys. Chem. A 118, 11943 (2014).

[90] A. I. Krylov, The quantum chemistry of open-shell species, in Reviews in Comp. Chem., edited by A. L. Parrill and K. B. Lipkowitz, volume 30, pages 151-224. J. Wiley \& Sons, 2017.

[91] M.J. Seaton, The hartree-fock equations for continuous states with applications to electron excitation of the ground configuration terms of oi, Philos. Trans. (London) 245, 469 (1953).

[92] A. F. White, M. Head-Gordon, and C. W. McCurdy, Complex basis functions revisited: Implementation with applications to carbon tetrafluoride and aromatic $\mathrm{N}$-containing heterocycles within the static-exchange approximation, J. Chem. Phys. 142, 054103 (2015).

[93] M. S. Szczygieł, M. Lesiuk, and R. Moszynski, Theoretical description of the ionization processes with a 
discrete basis set representation of the electronic continuum, arXiv:1909.07833 (2019).

[94] W. H. Miller, C. A. Slocomb, and H. F. Schaefer III, Molecular autoionization lifetimes and cross sections for Penning ionization: numerical results for $\mathrm{He}^{*}\left(1 s 2 s{ }^{3} \mathrm{~S}\right)+\mathrm{H}\left(1 s^{2} \mathrm{~S}\right)$, J. Chem. Phys. 56, 1347 (1972).

[95] Y. Shao, Z. Gan, E. Epifanovsky, A.T.B. Gilbert, M. Wormit, J. Kussmann, A.W. Lange, A. Behn, J. Deng, X. Feng, D. Ghosh, M. Goldey, P.R. Horn, L.D. Jacobson, I. Kaliman, R.Z. Khaliullin, T. Kus, A. Landau, J. Liu, E.I. Proynov, Y.M. Rhee, R.M. Richard, M.A. Rohrdanz, R.P. Steele, E.J. Sundstrom, H.L. Woodcock III, P.M. Zimmerman, D. Zuev, B. Albrecht, E. Alguires, B. Austin, G.J.O. Beran, Y.A. Bernard, E. Berquist, K. Brandhorst, K.B. Bravaya, S.T. Brown, D. Casanova, C.-M. Chang, Y. Chen, S.H. Chien, K.D. Closser, D.L. Crittenden, M. Diedenhofen, R.A. DiStasio Jr., H. Do, A.D. Dutoi, R.G. Edgar, S. Fatehi, L. Fusti-Molnar, A. Ghysels, A. Golubeva-Zadorozhnaya, J. Gomes, M.W.D. Hanson-Heine, P.H.P. Harbach, A.W. Hauser, E.G. Hohenstein, Z.C. Holden, T.-C. Jagau, H. Ji, B. Kaduk, K. Khistyaev, J. Kim, J. Kim, R.A. King, P. Klunzinger, D. Kosenkov, T. Kowalczyk, C.M. Krauter, K.U. Laog, A. Laurent, K.V. Lawler, S.V. Levchenko, C.Y. Lin, F. Liu, E. Livshits, R.C. Lochan, A. Luenser, P. Manohar, S.F. Manzer, S.-P. Mao, N. Mardirossian, A.V. Marenich, S.A. Maurer, N.J. Mayhall, C.M. Oana, R. Olivares-Amaya, D.P. O'Neill, J.A. Parkhill, T.M. Perrine, R. Peverati, P.A. Pieniazek, A. Prociuk, D.R. Rehn, E. Rosta, N.J. Russ,
N. Sergueev, S.M. Sharada, S. Sharmaa, D.W. Small, A. Sodt, T. Stein, D. Stuck, Y.-C. Su, A.J.W. Thom, T. Tsuchimochi, L. Vogt, O. Vydrov, T. Wang, M.A. Watson, J. Wenzel, A. White, C.F. Williams, V. Vanovschi, S. Yeganeh, S.R. Yost, Z.-Q. You, I.Y. Zhang, X. Zhang, Y. Zhou, B.R. Brooks, G.K.L. Chan, D.M. Chipman, C.J. Cramer, W.A. Goddard III, M.S. Gordon, W.J. Hehre, A. Klamt, H.F. Schaefer III, M.W. Schmidt, C.D. Sherrill, D.G. Truhlar, A. Warshel, X. Xu, A. Aspuru-Guzik, R. Baer, A.T. Bell, N.A. Besley, J.D. Chai, A. Dreuw, B.D. Dunietz, T.R. Furlani, S.R. Gwaltney, C.-P. Hsu, Y. Jung, J. Kong, D.S. Lambrecht, W.Z. Liang, C. Ochsenfeld, V.A. Rassolov, L.V. Slipchenko, J.E. Subotnik, T. Van Voorhis, J.M. Herbert, A.I. Krylov, P.M.W. Gill, and M. Head-Gordon, Advances in molecular quantum chemistry contained in the Q-Chem 4 program package, Mol. Phys. 113, 184 (2015).

[96] A. I. Krylov and P. M. W. Gill, Q-Chem: An engine for innovation, WIREs: Comput. Mol. Sci. 3, 317 (2013).

[97] E. Epifanovsky, M. Wormit, T. Kuś, A. Landau, D. Zuev, K. Khistyaev, P. U. Manohar, I. Kaliman, A. Dreuw, and A. I. Krylov, New implementation of high-level correlated methods using a general blocktensor library for high-performance electronic structure calculations, J. Comput. Chem. 34, 2293 (2013).

[98] M. Head-Gordon and J. A. Pople, A method for twoelectron Gaussian integral and integral derivative evaluation using recurrence relations, J. Chem. Phys. 89, 5777 (1988). 